\title{
Comparaison de deux systèmes d'élevage biologique d'ovins allaitants différant par le rythme de reproduction : une approche expérimentale pluridisciplinaire
}

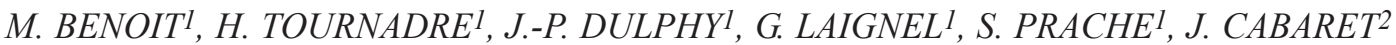 \\ ${ }^{1}$ INRA, UR1213 Herbivores, F-63122 Saint-Genès Champanelle, France \\ 2 INRA, UR1282 Infectiologie Animale et Santé Publique, F-37380 Nouzilly, France \\ Courriel : Marc.Benoit@clermont.inra.fr
}

En 2007, 1'élevage ovin allaitant en Agriculture Biologique (AB) représentait $1,8 \%$ du cheptel ovin viande en France, une proportion plus faible que pour les ovins lait $(3,9 \%)$ mais du même ordre de grandeur que celle observée pour les bovins, lait ou viande $(1,5 \%)$ (Agence Bio 2007). Cette faible proportion, par ailleurs en baisse entre 2006 et 2007 (- 6\% de brebis en $\mathrm{AB})$, peut être expliquée par $i$ ) de fortes contraintes liées à l'alimentation (importance et coût élevé des concentrés), ii) une maîtrise sanitaire souvent difficile, en particulier vis-à-vis des endoparasites comme les helminthes (Hovi et al 2003), iii) une structuration insuffisante du marché (Nardone et al 2004). Nos recherches, qui visent à développer une production ovine allaitante biologique durable ont ainsi croisé les regards de différentes disciplines pour aborder ces questions, en particulier celles relatives à l'élevage, l'alimentation, la maîtrise sanitaire, le type de produits et leur qualité, et les conditions d'une rentabilité économique suffisante.

Nous avons mis en place en domaine expérimental deux systèmes d'élevage différant par le rythme de reproduction des brebis, avec l'objectif d'évaluer leurs performances techniques et économiques et d'identifier les déterminants et limites de leur réussite. Ces systèmes ont visé un résultat économique élevé, avec deux stratégies contrastées construites autour de 2 combinaisons du niveau des charges opérationnelles et $\mathrm{du}$ niveau de la productivité numérique par brebis : dans le premier système, les brebis ont mis bas une fois par an (1/an), avec une proportion comparable d'agnelages au printemps et à l'automne, la stratégie globale étant de conjuguer une autonomie alimentaire élevée avec une large répartition des ventes d'agneaux au cours de l'année. Dans le second système, les brebis ont agnelé trois fois en deux ans (3en2), rythme qui a montré son intérêt économique en élevage conventionnel par rapport à un seul agnelage par an, via l'obtention d'une productivité numérique par brebis élevée (Speedy et FitzSimons 1977, Brelurut 1987, Benoit et al 1999, Benoit et Veysset 2003). Le système 3 en 2 est classiquement basé sur trois périodes de reproduction par an, souvent avec une synchronisation des brebis par un traitement hormonal pour la lutte de printemps. Ce système est plus exigeant pour les brebis. Il nécessite également un plus fort recours aux aliments concentrés et il est plus complexe à organiser que le système 1/an (Cournut et Dedieu 2004). Comme la règlementation $\mathrm{AB}$ interdit l'utilisation d'hormones de synthèse et limite l'utilisation des aliments concentrés et des molécules de synthèse (traitements allopathiques), nous avons testé l'hypothèse selon laquelle le système d'élevage 3en2 serait plus risqué en $\mathrm{AB}$ en termes de performances et de santé animale, ainsi que de rentabilité économique, et donc finalement serait moins durable que le système $1 / a n$.

\section{1 / Matériel et méthodes}

\section{1 / Dispositif expérimental}

a) Le site expérimental et la conduite des troupeaux

Cette étude a été réalisée de 2000 à 2003 à l'INRA de ClermontFerrand/Theix, sur la ferme expérimentale de Redon, située entre 700 et $850 \mathrm{~m}$ d'altitude sur des sols granitiques peu profonds. Les précipitations annuelles sont en moyenne de $700 \mathrm{~mm}$ avec de fréquentes sécheresses en été.

Le dispositif a comparé deux systèmes d'élevage, chacun comprenant 118 brebis et 24 ha, avec un chargement de $0,8 \mathrm{UGB} /$ ha de surface fourragère. Dans le système 1/an, les mises bas ont été réparties pour moitié en mars et pour l'autre moitié en novembre, cette répartition permettant i) d'optimiser la productivité des brebis, les femelles vides pouvant être recyclées d'une période sur l'autre, ii) de gérer précisément le renouvellement des animaux, avec une première mise bas des agnelles à 18 mois, iii) d'optimiser la période de vente des agneaux, avec une partie des ventes en hiver, période où les cours sont généralement élevés. Dans le système 3en2, les brebis, réparties en deux lots, ont mis bas successivement en mars, novembre et juin, avec un intervalle de huit mois entre mises bas (Benoit 1998). La mise en marché des agneaux était ainsi répartie sur toute l'année. 
Les surfaces expérimentales (48 ha, $52 \%$ de prairies fauchables, $27 \%$ de pâtures, $21 \%$ de parcours) ont été affectées de manière similaire entre les deux systèmes. Un mélange de céréales (triticale, orge, avoine) et de pois a été semé afin d'accrôitre l'autonomie alimentaire, avec la fourniture de 40 à $50 \%$ du concentré nécessaire $(1,1$ et 1,5 ha en $1 / a n$ et $3 e n 2$ respectivement). La rotation culturale était basée sur trois années de culture de céréalespois puis trois années de prairies temporaires. La conversion du site expérimental a débuté en 2000 ; la labellisation $\mathrm{AB}$ a été effective au 1 er janvier 2002, et la première vente d'agneaux sur la filière $A B$ a concerné ceux qui sont nés à l'automne 2001.

Les deux troupeaux expérimentaux ont été constitués à partir d'une troupe déjà présente sur le domaine et conduite sur le rythme d'un agnelage par an jusqu'en 1999. La transition entre la conduite précédente et celles des systèmes expérimentés (1/an et 3en2) s'est effectuée au cours de l'année 2000, et nous avons obtenu un fonctionnement stable des deux systèmes à partir de l'année 2001 (figures 1a et 1b). Les brebis, de race Limousine, ont été réparties dans les 2 troupeaux de façon homogène, selon leur poids et état corporel, leurs performances de reproduction précédentes (fertilité et prolificité), et en respectant une même pyramide d'âge.

b) Gestion des animaux, des prairies, des cultures, et de la fertilisation

La mise à l'herbe des brebis a été réalisée entre le 15 mars et le 5 avril, selon l'année. A cette période, lorsque les conditions climatiques étaient défavorables, les brebis allaitantes et leurs agneaux étaient rentrés en bergerie pour la nuit, les autres lots d'animaux restant au pâturage. En automne, les brebis gravides ont été rentrées en bergerie deux semaines avant le début des mises bas, les autres brebis restant au pâturage jusqu'à la mi-décembre. En bergerie, les brebis en fin de gestation et en allaitement étaient réparties en deux groupes selon leur stade de gestation ou leur date de mise bas, de manière à ajuster les apports d'aliments concentrés. Pour ces brebis, le foin était offert à volonté, tandis que l'enrubannage était limité pour respecter le cahier des charges de l'agriculture biologique (au maximum 50\% de la matière sèche de la ration offerte). Les quantités d'aliments concentrés distribuées aux brebis (mélange de céréales et pois produits, céréales et complémentaire azoté achetés) étaient calculées à partir des recommandations INRA, soit en moyenne $400 \mathrm{~g} / \mathrm{j}$ et par brebis sur les 6 dernières semaines de gestation, et $500 \mathrm{~g} / \mathrm{j}$ et par brebis au cours des 10 premières semaines de lactation. En $3 e n 2$, les brebis agnelant en juin ne

Figure 1a. Fonctionnement de la reproduction (luttes et mises bas) et productivité numérique par brebis.

Système 1/an (1 agnelage par an).

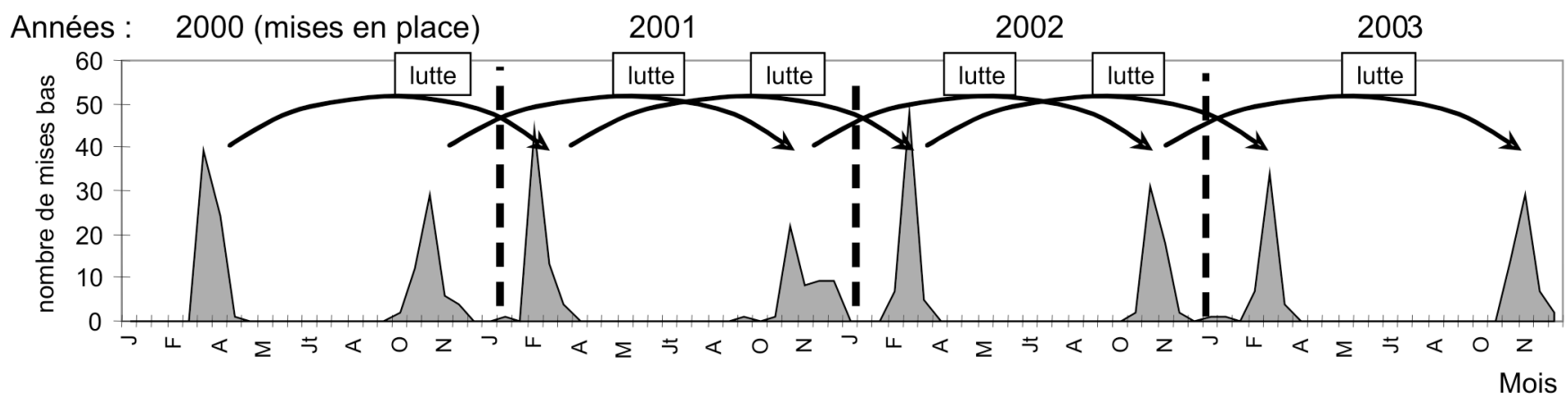

$\%$ des mises bas annuelles

55

45

53

57

57

53

Productivité numérique du troupeau

1,43

1,52

1,58

Figure $1 \mathrm{~b}$. Fonctionnement de la reproduction (luttes et mises bas) et productivité numérique par brebis.

Système 3en2 (3 agnelages en 2 ans).

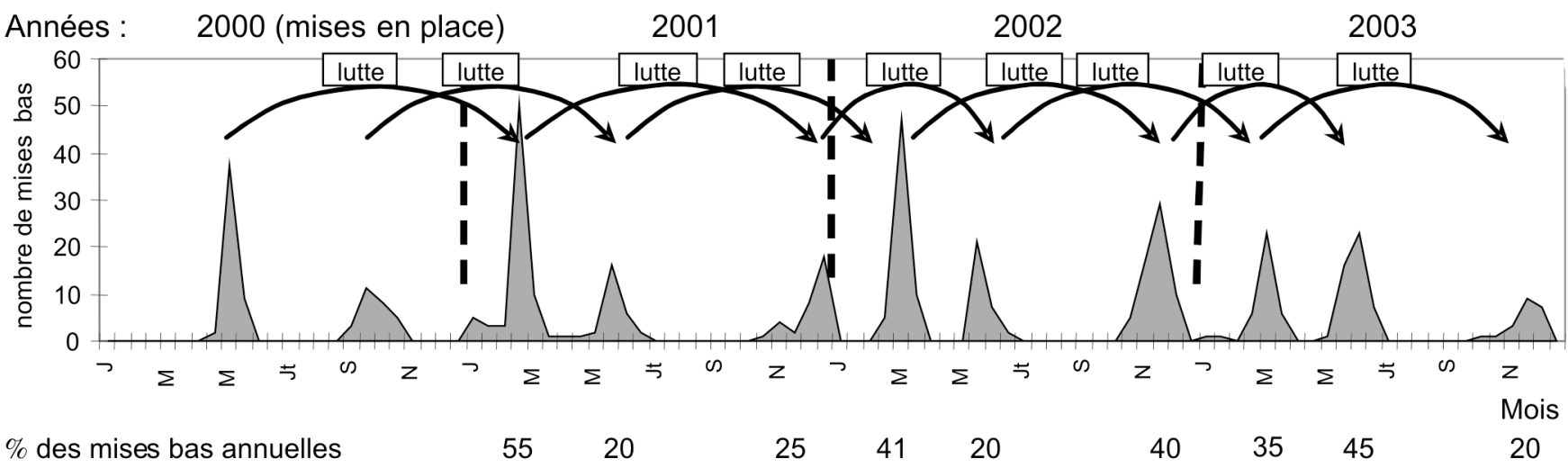

Productivité numérique du troupeau 
recevaient pas de concentré. Pour les deux systèmes, les agneaux ne pouvant être allaités par leur mère ont été sortis de l'élevage et considérés vendus au prix de $10 €$. En 1/an, les agneaux nés en mars ont été allaités par leur mère aussi longtemps que la qualité du pâturage le permettait, tel que recommandé par Prache et al (1986) ; ils ont en moyenne été sevrés à l'âge de $84 \mathrm{j}$. Les agneaux nés en novembre ont été sevrés en moyenne à l'âge de $78 \mathrm{j}$. En 3en2, les agneaux ont été sevrés plus jeunes, de manière à limiter la mobilisation des réserves corporelles des mères et éviter de pénaliser la réussite de la reproduction suivante (Cognié et al 1984). Ainsi, les âges moyens au sevrage ont été de 58,71 et $67 \mathrm{j}$ pour les agneaux nés respectivement en mars, juin et novembre. Dans les deux systèmes, les agneaux nés en novembre ont été nourris en bergerie avec du concentré et du foin, le concentré étant limité à $600 \mathrm{~g} / \mathrm{j}$ par agneau, en conformité avec le règlement de l'agriculture biologique $(40 \%$ de concentré dans la ration). Les agneaux nés en mars et en juin ont été élevés au pâturage. En $3 e n 2$, les agneaux ont été complémentés au pâturage depuis la naissance jusqu'à l'abattage ; le concentré était offert ad libitum jusqu'à hauteur de $600 \mathrm{~g} / \mathrm{j}$ par agneau. En 1/an, les agneaux d'herbe n'ont pas reçu de complémentation avant le sevrage ; ensuite, si un complément était nécessaire, ils ont été complémentés ad libitum jusqu'à hauteur de $600 \mathrm{~g} / \mathrm{j}$ par agneau selon la disponibilité en herbe de qualité. Les agneaux ont été abattus à l'abattoir expérimental situé à $6 \mathrm{~km}$ de la ferme, lorsqu'ils avaient

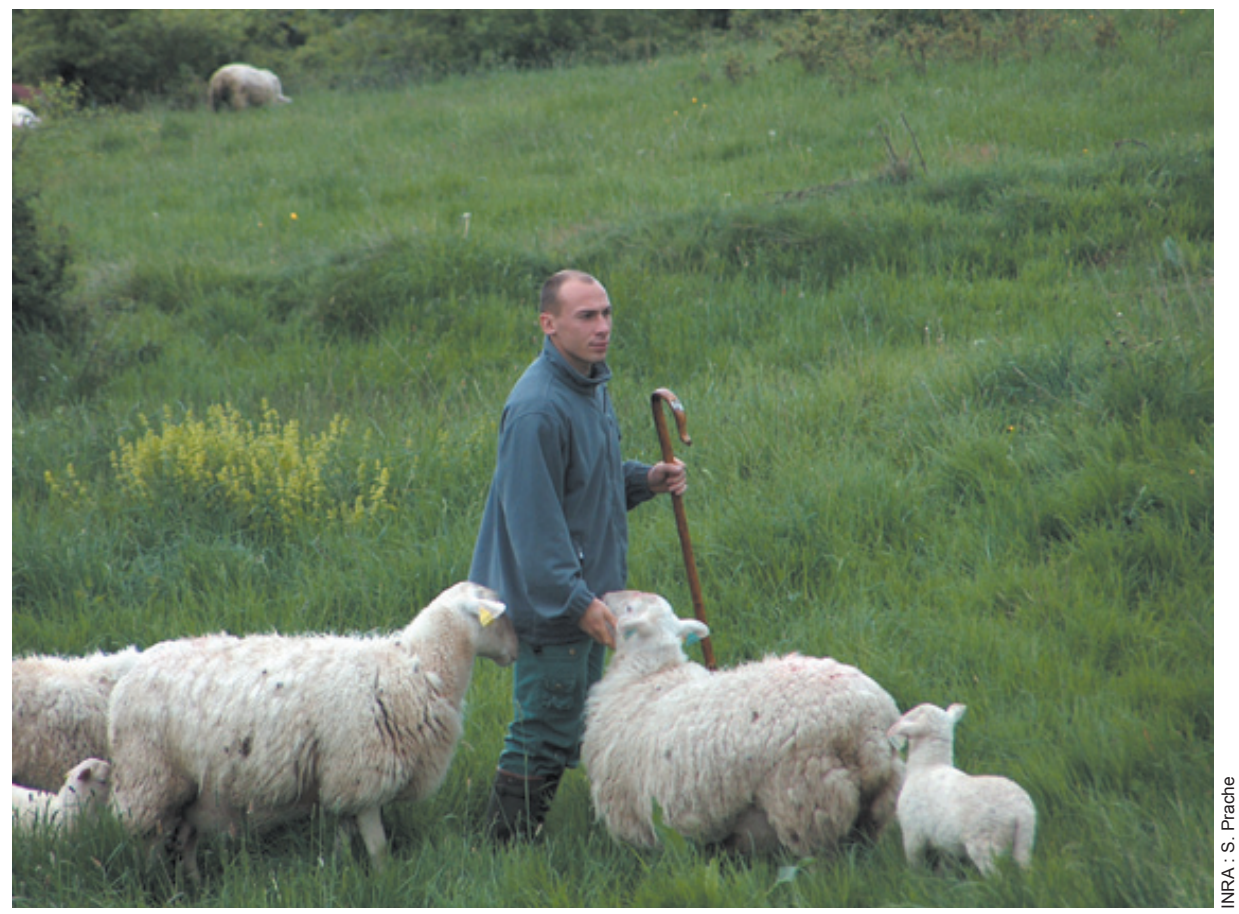
faisant. A partir de début 2002 (fin de période de conversion), les agneaux présentant des notes insatisfaisantes de conformation ( $\mathrm{P}$; grille EUROP) ou d'état d'engraissement (note 4) ont été exclus de la certification par la coopérative. Conformément à ces critères, $67 \%$ et $55 \%$ des agneaux 1/an ont été certifiés AB en 2002 et 2003 respectivement. Ces proportions étaient similaires en $3 e n 2$ (64\% en 2002 et $60 \%$ en 2003).

c) Production fourragère et qualité des fourrages

Dans la mesure du possible, les prairies ont été fauchées précocement et le fourrage a été enrubanné $(30 \%$ du total des fourrages conservés). Nous avons considéré que l'azote était fourni par les légumineuses pour les surfaces fourragères, et par les protéagineux pour les cultures (Doyle et Topp 2004). Le compost des litières a représenté $125 \mathrm{~kg}$ Matière Sèche (MS) (35\% MS) par brebis et par an, avec une composition $\mathrm{N} / \mathrm{P} / \mathrm{K}$ de $24 / 8 / 43$ (g/kg MS). Il a été épandu sur les prairies fauchées et sur les surfaces en céréales en deuxième et troisième année, à raison de $5 \mathrm{~T} / \mathrm{ha}$. Le chargement et la conduite du pâturage ont été comparables dans les deux systèmes. La production fourragère et la qualité des fourrages ont été mesurées sur l'ensemble des surfaces des deux systèmes, sans les distinguer. La production fourragère a atteint $6,4 \mathrm{~T} \mathrm{MS} / \mathrm{ha}$ en 2000 et 2001. Elle a fortement diminué en 2002 (4,2 T MS/ha) et en 2003 (3,8 T $\mathrm{MS} / \mathrm{ha}$ ) en raison d'une sécheresse sévère. En moyenne au cours de la période atteint un niveau d'engraissement satis- expérimentale, la valeur énergétique du foin a été de 0,65 UFL (INRA 1989) et sa teneur en protéines brutes de $91 \mathrm{~g} / \mathrm{kg}$ MS ; cette dernière a diminué au cours de la période expérimentale, de 101 à 82 $\mathrm{g} / \mathrm{kg} \mathrm{MS}$, en relation avec une baisse de la proportion des légumineuses, dont la note d'abondance (cf. $\S 1.2$ ) est passée de 2,5 à 1,4 sur les prairies et de 1,6 à 1,3 sur les parcours. Il faut cependant noter que la production fourragère et la teneur en protéines se sont rétablies en 2004 $(6,5 \mathrm{~T} \mathrm{MS} / \mathrm{ha}$ et $110 \mathrm{~g} / \mathrm{kg} \mathrm{MS})$ du fait de meilleures conditions climatiques. La production de pois à été très faible lors de 3 des 4 années expérimentales (problème de gel).

\section{d) Gestion du parasitisme}

La stratégie de prévention du parasitisme a été la suivante : la contamination des parcelles a été contenue, en autorisant un seul pâturage par les brebis en lactation (suivi d'une fauche ou d'un pâturage par des brebis taries), et en effectuant un traitement anthelminthique sur les brebis avant la mise à l'herbe (Athanasiadou et al 2007). L'infestation des agneaux après le sevrage a été limitée en les engraissant sur des parcelles «propres», préalablement fauchées. Nous avons utilisé des produits allopathiques de synthèse pour les traitements anthelminthiques, car l'évaluation de traitements alternatifs est seulement en cours et nous n'aurions pas contrôlé de manière sûre les incidents pathologiques ou parasitaires (Cabaret et al 2002).

\section{e) Gestion de la reproduction}

Les mêmes béliers ont été utilisés dans les deux troupeaux (race Limousine pour le renouvellement des femelles et race Ile-de-France pour les agneaux commercialisés). Lors de la lutte du mois d'octobre, les béliers ont été maintenus avec les femelles pendant $35 \mathrm{j}$ dans les deux systèmes. Lors de la lutte de juin, lorsque l'activité sexuelle de la race Limousine est faible (Walrave et al 1975, Tournadre et al 2002), l'effet bélier a été utilisé dans les deux systèmes pour induire une activité sexuelle chez les brebis précédemment en anoestrus (Signoret 1990, Thimonier et al 2000). Les béliers ont été introduits de fin mai à mi-juillet (3en2) ou à fin juillet (1/an), comme recommandé par Tournadre et al (2002). Dans le 3en2, la troisième période de lutte qui a eu lieu avant l'entrée des brebis en anoestrus (Walrave et al 1975) a débuté le 20 décembre et a duré $35 \mathrm{j}$. Dans le système 1/an les brebis étaient réparties en deux lots correspondant aux deux périodes de 
lutte (figure 1a). Les intervalles entre la mise bas et la mise en reproduction ont été de $208 \pm 17 \mathrm{j}$ lors des luttes d'octobre et de $197 \pm 7 \mathrm{j}$ lors des luttes de juin. Dans le système 3en 2 (figure 1b), les brebis étaient aussi réparties en deux lots dont les luttes étaient espacées d'environ 8 mois (Marzin et Brelurut 1979). Ainsi, les intervalles entre la mise bas et la mise en reproduction ont été de $136 \pm 12$ j lors des luttes d'octobre et de $88 \pm 8 \mathrm{j}$ lors des luttes de juin. Lors des luttes de janvier, cet intervalle a été de $76 \mathrm{j}$ en 2000 (période de transition) puis de $12 \mathrm{j}$ en 2001 et $32 \mathrm{j}$ en 2002. En conséquence, les brebis luttées en janvier 2001 et 2002 étaient encore allaitantes.

Pour les deux systèmes, les brebis vides lors d'une session de lutte étaient remises à la reproduction en même temps que l'autre moitié du troupeau, ou réformées. Les agnelles de renouvellement étaient issues des différentes périodes d'agnelage de chaque système et le nombre conservé était calculé en fonction des prévisions de réforme. Les agnelles nées en mars étaient mises à la reproduction en janvier ( 3 en2) ou juin (1/an) de l'année suivante, celles nées en novembre (1/an et 3en2) et juin (3en2) l'étaient respectivement en octobre et juin de l'année suivante. En 1/an, le principe a été de mettre en lutte au printemps les deux tiers de l'ensemble des agnelles conservées, afin d'atteindre l'équilibre de $50 \%$ des mises bas du troupeau au printemps et à l'automne.

\section{2 / Mesures}

a) Fourrages : production, qualité, consommation et indices de nutrition

Dans les prairies fauchées, la production de fourrage a été estimée à partir du comptage des balles récoltées et de la pesée de balles choisies au hasard. Dans les parcelles pâturées, la production de fourrage a été estimée au moyen de 24 cages de mise en défens disposées dans 12 parcelles représentatives, dans chaque système. Des fauches sous cages ont été réalisées en mai, juillet et septembre. Sur des échantillons de foin prélevés au hasard sur les balles, nous avons mesuré la teneur en protéines brutes (CP, Dumas N x 6,25), en cellulose brute et la digestibilité cellulase (Aufrère et Michalet-Doreau 1988), pour estimer l'énergie nette (INRA 1989). L'abondance des légumineuses a été évaluée visuellement en juillet en utilisant une échelle de 0 à 5 , la note 0 correspondant à l'absence de légumi- neuses et la note 5 à une proportion de $75 \%$. L'ingestion d'herbe par les animaux au pâturage a été estimée à partir du nombre d'animaux présents et de la durée de leur séjour, en faisant l'hypothèse d'une ingestion quotidienne de $1,7 \mathrm{~kg}$ MS pour les brebis taries et en gestation, 3,0 kg MS pour les brebis allaitantes, 2,0 kg MS pour les béliers et $1,0 \mathrm{~kg}$ MS pour les agneaux sevrés (Thériez 1983). Les indices de nutrition des fourrages ont été calculés selon Salette et Lemaire (1981) pour l'indice de nutrition azotée, et Salette et Huche (1991) pour les indices P et K. Ils n'ont pas été évalués en 2003, la production fourragère ayant été trop faible.

\section{b) Performance des animaux}

Les agneaux ont été pesés à la naissance puis à 10,30 et $70 \mathrm{j}$ d'âge, au sevrage et à l'abattage. Le poids vif des agneaux et leur vitesse de croissance ont fait l'objet d'une analyse de variance (SAS 1999) afin d'analyser l'effet du système de production, de la période de mise bas et de l'interaction entre ces facteurs, en prenant en compte l'effet du sexe, de la race et du poids de naissance. La productivité numérique par brebis a été calculée comme étant le nombre d'agneaux produits par brebis de plus de un an et par an. L'effet du système de production sur la fertilité et la prolificité a été analysé par test du $\chi^{2}$.

\section{c) Qualité de la carcasse}

Les mesures ont été effectuées en 2001, 2002 et 2003, à $24 \mathrm{~h}$ de ressuyage après l'abattage. La carcasse a été pesée et sa conformation classée sur une échelle de 0 à 15 telle que décrite par Fisher et al (2000). L'état d'engraissement a été évalué par le poids du gras périrénal. La couleur (indices L*, a* et b*) du gras sous-cutané caudal a été mesurée avec un spectrocolorimètre MINOLTA CM-2002 (illuminant $\mathrm{D}_{65}$ ). La fermeté du gras sous-cutané dorsal a été évaluée manuellement sur une échelle allant de 3 «gras huileux» à 15 «gras dur». L'effet du système de production sur les caractéristiques de la carcasse a été analysé par analyse de variance (SAS 1999), en tenant compte des effets du sexe et de l'année.

\section{d) Santé des animaux}

La mortalité des brebis et des agneaux a été enregistrée, ainsi que les causes probables de mortalité et de réforme des brebis. Nous avons mesuré l'infestation par les strongles gastrointestinaux et Moniezia par comptage des œufs émis par gramme de fèces (Bentounsi et al 2007). Des échantillons de fèces ont été prélevés tous les mois entre mars et novembre sur cinq brebis et cinq agneaux par système, choisis au hasard au début de la saison de pâturage (remplacement au hasard en cas de sortie du dispositif). Pour connaître le niveau initial de parasitisme et les types de parasites présents sur le site expérimental, des autopsies ont été réalisées à l'automne précédant la conversion à l'AB sur quatre brebis et cinq agneaux, puis ensuite chaque année sur trois agneaux et trois brebis de chaque système. Ces animaux ont été choisis au hasard à la fin de la saison de pâturage pour les agneaux et, pour les brebis, parmi celles réformées pour cause de problème de reproduction ou d'âge. La caillette, l'intestin grêle et le gros intestin ont été traités selon les procédures du MAAF (1986). Les nématodes ont été dénombrés par espèce présente. Les protostrongles ont été recherchés dans les poumons des brebis (Cabaret et al 1980). A l'automne 2000, nous avons recherché Oestrus ovis dans les sinus des agneaux selon la méthode de Yilma et Dorchies (1991). La mortalité des brebis et des agneaux a été analysée par le test exact de Fisher pour déterminer les différences entre systèmes d'élevage. De même, le nombre d'œufs par gramme de fèces et les résultats d'autopsies ont été analysés par analyse de variance après transformation logarithmique afin de stabiliser la variance (SAS 1999), ceci pour déterminer l'effet du système d'élevage et du type d'animal (brebis vs agneaux).

\section{e) Résultats économiques}

Les définitions des critères et les méthodes de calcul utilisées sont détaillées par Benoit et Laignel (2006). La marge brute par brebis est la différence entre le produit brut ovin et les charges opérationnelles de l'atelier ovin, le produit brut étant la somme des recettes des ventes des animaux, des recettes diverses (laine, en particulier) et des aides ovines, déduction faite des achats d'animaux; les charges de l'atelier ovin comprennent les achats d'aliments, d'engrais et de semences fourragères, les cessions internes de céréales produites sur la ferme, ainsi que les frais vétérinaires et d'élevage. L'autonomie alimentaire a été calculée comme suit : [(Besoins en Energie du Troupeau (BET)) - (énergie apportée par les aliments achetés)] / (BET). Le même type de ratio a été calculé pour l'autonomie fourragère : [BET - (énergie des aliments achetés et des concen- 
Tableau 1. Performances de reproduction.

\begin{tabular}{|c|c|c|c|c|c|}
\hline Année & $2000^{2}$ & 2001 & 2002 & 2003 & $\begin{array}{l}\text { Moyenne } \\
2001-2003\end{array}$ \\
\hline $1 / a n^{7}$ & & & & & \\
\hline Fertilité \% & 81 & 91 & 94 & 95 & 93,4 \\
\hline Prolificité $\%$ & 158 & 160 & 166 & 177 & 165,8 \\
\hline Mortalité des agneaux \% & 12 & 14 & 12 & 11 & 12,3 \\
\hline Taux de mise bas $\%$ & 97 & 104 & 106 & 101 & 104 \\
\hline Productivité Numérique \% & (nc) & 143 & 152 & 158 & 151 \\
\hline 3en $2^{1}$ & & & & & \\
\hline Fertilité \% & 60 & 75 & 77 & 67 & 73,1 \\
\hline Prolificité \% & 156 & 153 & 153 & 158 & 152,2 \\
\hline Mortalité des agneaux \% & 15 & 26 & 13 & 17 & 17,8 \\
\hline Taux de mise bas $\%$ & 69 & 134 & 144 & 109 & 129 \\
\hline Productivité Numérique \% & (nc) & 151 & 193 & 140 & 161,3 \\
\hline
\end{tabular}

1 1/an et 3en2 : un agnelage par brebis et par an et 3 agnelages en 2 ans, respectivement. (nc) non calculé, le taux de mise bas n'étant pas stabilisé en 3en2.

2 année 2000 : année de transition.

trés produits sur la ferme)] / (BET). L'énergie des aliments achetés et les BET ont été estimés à partir des tables INRA (1989). Ces données ont été comparées à celles obtenues dans 10 élevages privés $\mathrm{AB}$ et 16 élevages privés conventionnels suivis selon la même méthodologie (Laignel et Benoit 2004), l'objectif étant de situer le niveau de performance des deux systèmes d'élevage étudiés et d'identifier, sur ces références, les éléments déterminants permettant d'expliquer les résultats observés.

\section{2 / Résultats}

\section{1 / Performances de production et de reproduction (tableau 1)}

Sur la période 2001-2003, la fertilité a été plus favorable en 1/an qu'en 3en 2 : en moyenne, elle a été de 93,4\% en $1 /$ an vs $73,1 \%$ en $3 e n 2(\mathrm{P}<0,001)$. La fertilité du système 1/an a été stable au cours des 3 années expérimentales (figure 2) et élevée, quelle que soit la saison de mise à la reproduction

Figure 2. Fertilité des femelles selon la période de lutte, pour les systèmes 1/an et 3en2.

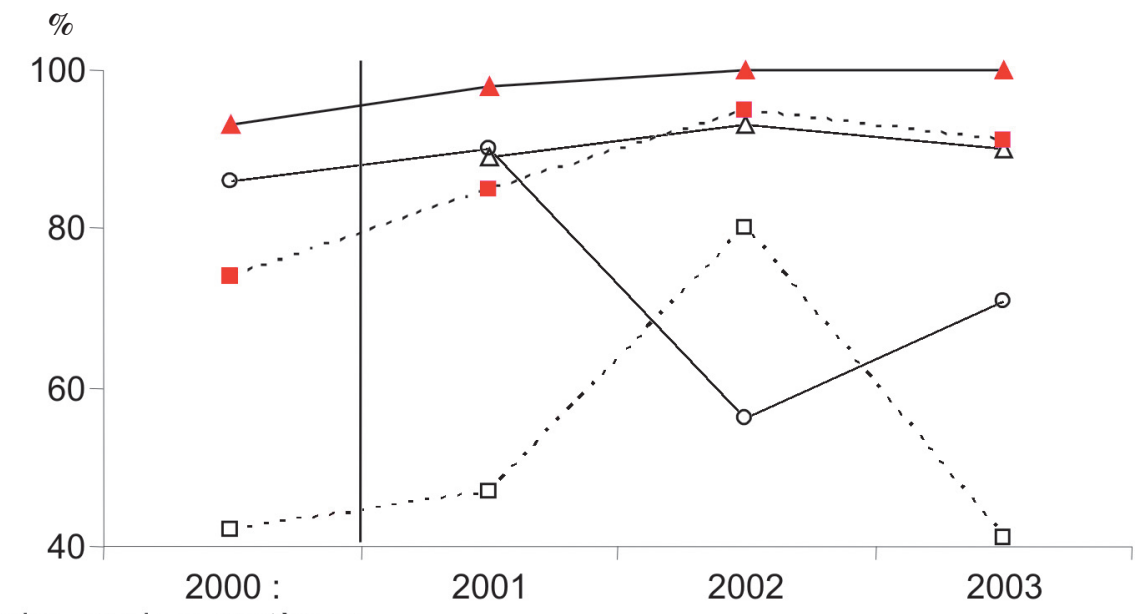

mise en place systèmes

\section{Année de mise bas}

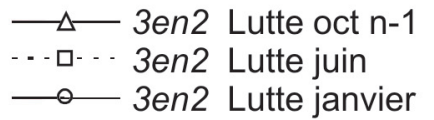

$(99,4 \%$ en octobre et $89,6 \%$ en juin), en comparaison de celle observée au cours des décennies précédentes sur la même troupe de brebis conduite en un agnelage par an (Brelurut 1987). Au contraire, la fertilité en $3 e n 2$ a été très variable selon les années et la saison de mise à la reproduction, sauf pour les luttes d'octobre. A cette période, l'intervalle entre la mise bas précédente et la lutte a peu varié entre années (136 \pm $11,7 \mathrm{j}$ ), et la fertilité a atteint en moyenne $91,7 \%$, résultat comparable à ceux obtenus par cette troupe en conduite accélérée (Brelurut 1987). En janvier, la fertilité a été satisfaisante en 2001 $(90 \%)$ car l'intervalle entre la mise bas précédente et la lutte était assez long (76 j). Inversement, en janvier 2002 et 2003 , lorsque cet intervalle était court (12 et $32 \mathrm{j}$ respectivement), la fertilité a été moindre $(55,6 \%$ en 2002 et $71,2 \%$ en 2003), en raison d'une fertilité plus faible des brebis qui étaient accélérées : $29,6 \%$ en 2002 et $71 \%$ en 2003 (figure 3$)$. Enfin, la fertilité en juin a été très faible en $2001(46,7 \%)$ et $2003(41,2 \%)$, mais satisfaisante en $2002(80,3 \%)$. Les raisons de ces variations annuelles sont diverses, liées à une fertilité médiocre des brebis en accélération $(57,9 \%$ en 2001 et $57,1 \%$ en 2003), des agnelles (fertilité nulle en 2001), ou des brebis vides de la lutte précédente (fertilité de $6,3 \%$ en 2003 , probablement en raison de l'exposition de ces animaux, et en particulier des béliers, à de très fortes températures). Les bons résultats de la lutte de juin 2002 ont été obtenus en cumulant des fertilités élevées pour les brebis accélérées $(82,7 \%)$ et les agnelles $(100 \%)$ et, dans une moindre mesure, pour les brebis vides de la lutte précédente $(70 \%)$. Il faut noter qu'à cette saison de reproduction, l'intervalle entre la mise bas précédente et la lutte a été assez constant au cours des trois années $(88 \pm$ $7,6 \mathrm{j})$.

La fertilité des agnelles a été plus élevée en 1/an qu'en $3 e n 2$ (90,3 vs 71,2\%, $\mathrm{P}<0,01)$, probablement parce que ces animaux étaient plus âgés (12,8 vs 10,4 mois, $\mathrm{P}<0,001)$ et plus lourds à la lutte $(47,5$ vs $44,4 \mathrm{~kg}, \mathrm{P}<0,01)$.

En système 1/an, la prolificité, en moyenne annuelle, a régulièrement augmenté entre 2001 et 2003 (figure 4). En système 3en2, la même évolution a été observée pour les luttes d'octobre, mais la prolificité a été beaucoup plus variable pour les mises en reproduction de janvier et de juin. Les niveaux de prolificité des deux systèmes n'ont pas été différents pour la lutte d'octobre : $169 \%$ en $3 e n 2$ contre $172 \%$ en $1 / a n$. 
Figure 3. Fertilité des différentes catégories de femelles composant les deux lots du système d'élevage 3en2 (3 agnelages en 2 ans), aux différentes périodes de mise à la reproduction.

Les nombres indiqués au dessus des barres des histogrammes correspondent aux effectifs de brebis mises en lutte.
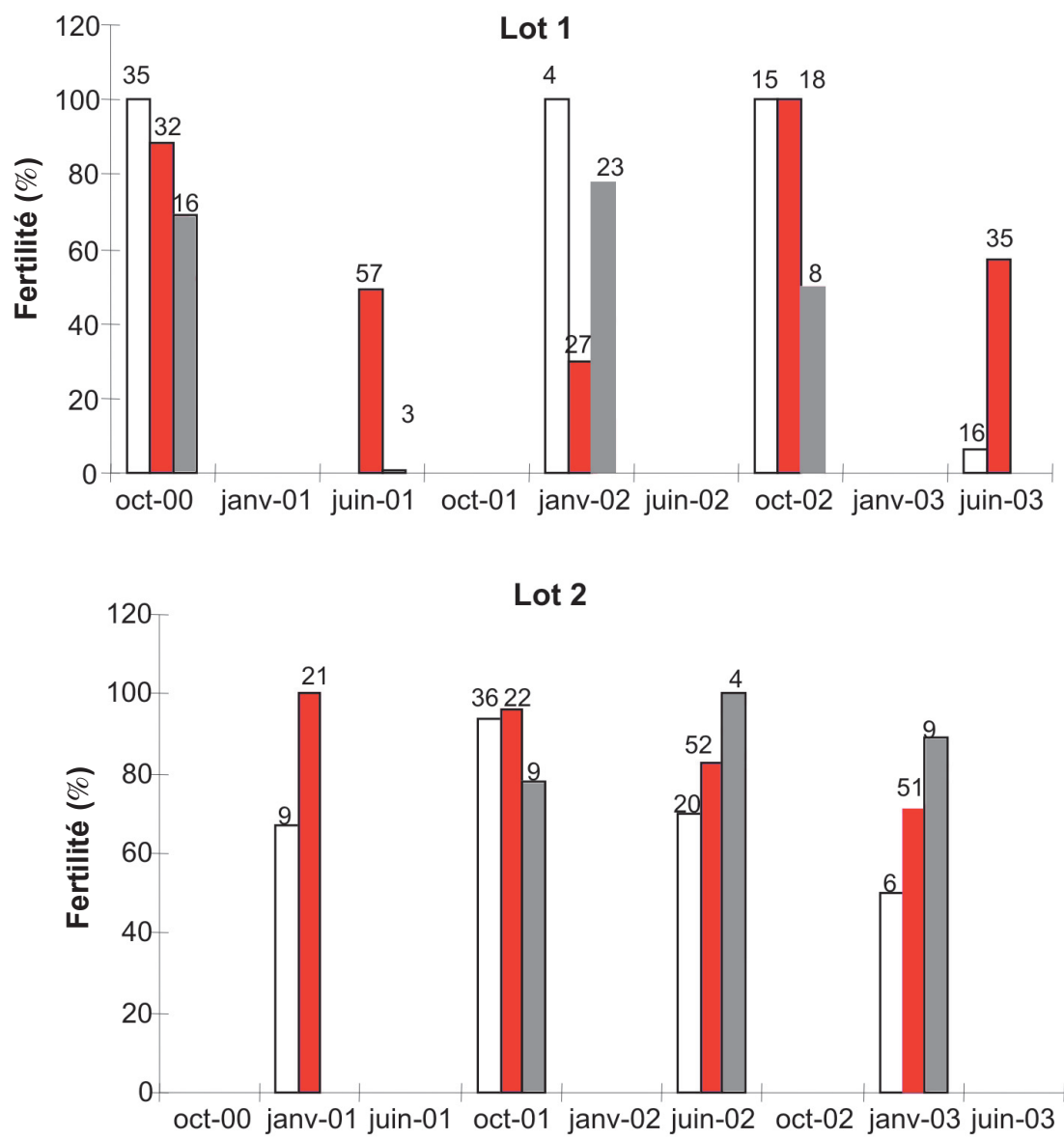

brebis vides à la lutte précédente

brebis accélérées

agnelles

Figure 4. Prolificité des femelles selon la période de mise bas, pour les systèmes 1/an et 3en2.

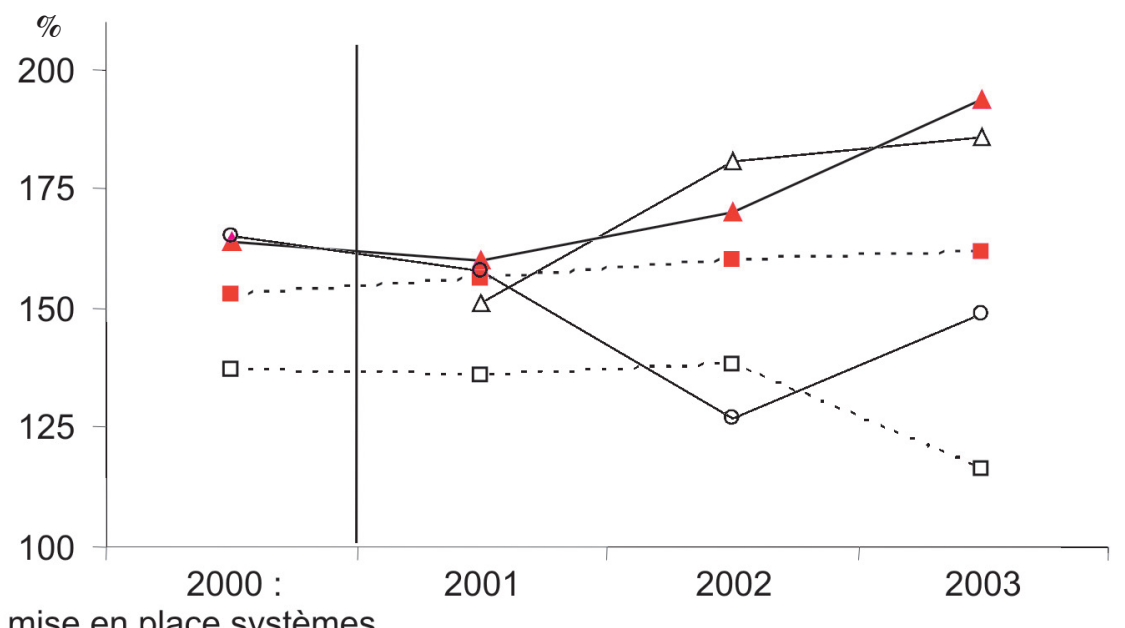

mise en place systèmes

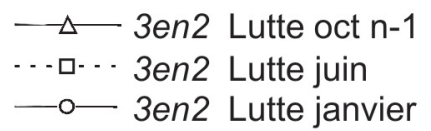

$\mathrm{Au}$ contraire, pour les luttes de juin, la prolificité a été plus faible en 3en2 qu'en 1/an : $133 \%$ contre $159 \%$ $(\mathrm{P}<0,001)$. Pour les luttes de janvier en $3 e n 2$, on observe des niveaux de prolificité intermédiaires $(145 \%)$ entre ceux des luttes d'octobre et de juin. Au final, la prolificité a globalement été plus faible en 3en2 qu'en 1/an (152 vs $166 \%, \mathrm{P}<0,05)$, comme déjà observé par Brelurut (1987).

Comme attendu, le taux de mise bas a été plus élevé en 3en 2 qu'en 1/an : sur la période 2001-2003, 33\% des brebis ont agnelé deux fois par an en $3 e n 2$ contre $4 \%$ en 1/an. En moyenne, la productivité numérique par brebis a été supérieure de $7 \%$ en 3 en 2 (161,3\% contre $151 \%$ en $1 /$ an), mais beaucoup plus variable, variant de $140 \%$ à $193 \%$ contre $143 \%$ à $158 \%$ en $1 /$ an (figure 5). Dans le système $3 e n 2$, la productivité numérique par brebis observée en 2002 a été considérée comme la plus élevée possible avec ce rythme de reproduction (taux de mise bas ponctuellement de 144\%)

\section{2 / Caractéristiques des agneaux et performances de croissance (tableau 2)}

Les agneaux nés simples, doubles et triples ont représenté respectivement $28 \%, 67 \%$ et $5 \%$ de l'ensemble des agneaux nés ; 6,3\% des agneaux n'ont pas pu être allaités par leur mère. Les agneaux engraissés à l'herbe ont représenté $73 \%$ du nombre total des agneaux en $3 e n 2$ et $50 \%$ en $1 / a n$.

La vitesse de croissance entre la naissance et l'âge de $70 \mathrm{j}$ (période d'allaitement) des agneaux nés en mars ( $234 \mathrm{~g} / \mathrm{j}$ en $3 e n 2$ et $248 \mathrm{~g} / \mathrm{j}$ en $1 / \mathrm{an}$ ) et en novembre $(243 \mathrm{~g} / \mathrm{j}$ en 3 en 2 et $263 \mathrm{~g} / \mathrm{j}$ en $1 / a n$ ) a été plus faible pour le système $3 e n 2(\mathrm{P}<0,01)$. Les agneaux nés en juin ont eu une vitesse de croissance plus faible que ceux nés en mars (204 vs $234 \mathrm{~g} / \mathrm{j}, \mathrm{P}<0,001)$.

Après le sevrage, la vitesse de croissance des agneaux nés en novembre et engraissés en bergerie a aussi été plus élevée en 1/an qu'en 3en2 (235 vs 212 $\mathrm{g} / \mathrm{j}, \quad \mathrm{P}<0,001)$, les quantités de concentré consommées étant peu différentes entre les deux systèmes $(50,2$ $\mathrm{kg}$ en $3 e n 2$ vs 46,1 $\mathrm{kg}$ en $1 / \mathrm{an}$ ). A l'inverse, la vitesse de croissance après le sevrage des agneaux nés en mars et engraissés à l'herbe a été plus élevée en $3 e n 2$ qu'en 1/an (201 vs $185 \mathrm{~g} / \mathrm{j}$, $\mathrm{P}<0,01)$, principalement en raison des différences de complémentation 
Figure 5. Productivité numérique par brebis et taux de doubles mises bas par année, pour les systèmes 1/an et 3en2.

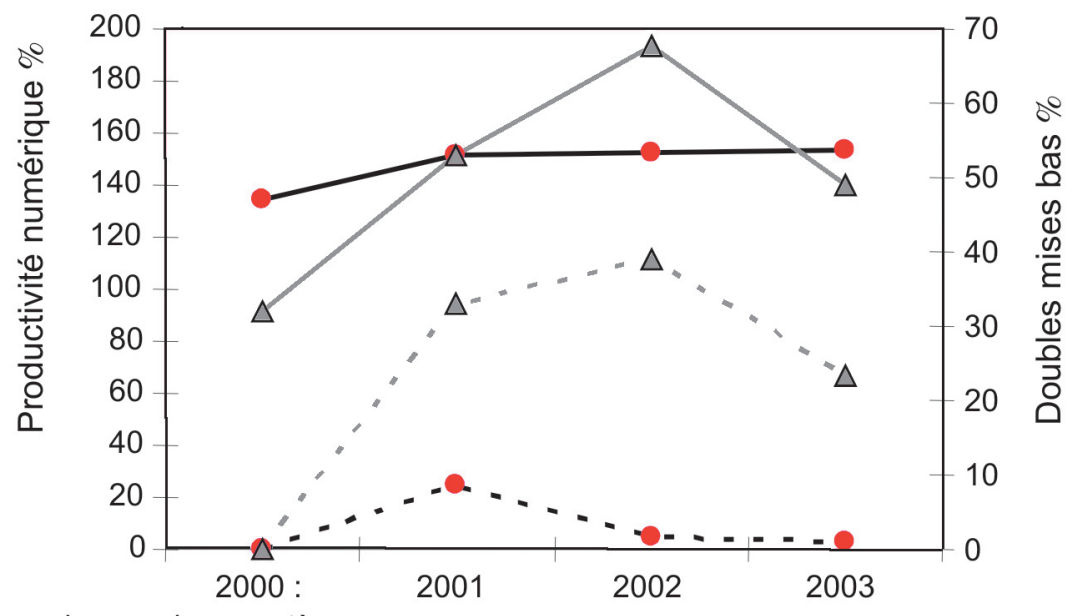

mise en place systèmes

$$
\begin{aligned}
& - \text { - 1/an : productivité numérique }-\triangle \text { 3en } 2 \text { : productivité numérique } \\
& --- \text { 1/an : doubles mises bas } \quad-\Delta^{-}-3 e n 2: \text { doubles mises bas }
\end{aligned}
$$

au pâturage. Malgré un apport de concentré au pâturage, les agneaux du système $3 e n 2$ nés en juin ont affiché une croissance inférieure à celle de ceux nés en mars (169 vs $201 \mathrm{~g} / \mathrm{j}$, $\mathrm{P}<0,001)$. Plusieurs explications peuvent être avancées. En premier lieu, il peut y avoir concurrence entre les agneaux nés en mars et ceux nés en juin pour les prairies de bonne qualité. De plus, le niveau de parasitisme est souvent le plus élevé pendant l'été, et le risque de contamination s'accroît avec la limitation des apports de concentré lorsque les disponibilités en herbe, de moindre qualité, se réduisent fortement (Prache et al 1992). Enfin, les fortes températures estivales peuvent diminuer la vitesse de croissance des agneaux.
L'âge et le poids vif au sevrage ont été inférieurs dans le système 3 en 2 , en lien avec les différences de conduite liées au rythme de reproduction des brebis. Ce poids plus faible au sevrage explique en partie la consommation de concentré plus élevée pour les agneaux au pâturage en 3en2 qu'en 1/an (42,9 vs $18,5 \mathrm{~kg})$, les apports de concentrés aux agneaux 3 en 2 étant par ailleurs systématiques. L'âge moyen à l'abattage n'a pas différé entre systèmes : il a été en moyenne de $149 \mathrm{j}$ pour les agneaux d'herbe et de $123 \mathrm{j}$ pour les agneaux de bergerie. Le poids vif à l'abattage des agneaux de bergerie a été un peu plus élevé en 1/an qu'en $3 e n 2$ $(+1,2 \mathrm{~kg}, \mathrm{P}<0,05)$. Pour ce critère, il n'y a pas eu de différence entre systèmes pour les agneaux d'herbe nés en mars $(34,9 \mathrm{~kg})$. En 3en2, les agneaux d'herbe nés en juin ont été abattus à un poids vif plus faible que ceux nés en mars (- $3 \mathrm{~kg}, \mathrm{P}<0,001)$.

\section{3 / Caractéristiques et qualité des carcasses (tableau 3)}

Au cours de la période 2001-2003, 356 et 396 agneaux ont été abattus dans les systèmes $1 /$ an et 3 en 2 respectivement. Le poids de la carcasse a été plus élevé en 1/an qu'en 3en2 (15,52 vs $15,08 \mathrm{~kg}, \mathrm{P}<0,05)$. L'état d'engraissement, la note de conformation, et la couleur du gras dorsal ont été similaires pour les deux systèmes. La fermeté du gras dorsal a été plus faible chez les agneaux 3en 2 que chez les agneaux 1/an (10,2 vs 11,1, $\mathrm{P}<0,001$.)

\section{4 / Santé animale}

\section{a) Indicateurs généraux}

Les proportions des principales pathologies chez les brebis (mammites, troubles digestifs ou troubles de la reproduction) n'ont pas été différentes entre les deux systèmes (tableau 4). La mortalité périnatale (jusqu'à $10 \mathrm{j}$ d'âge) a été plus élevée $(14,2 \%)$ dans le système 3en 2 que dans le système 1/an $(10 \%, \mathrm{P}<0,05)$, essentiellement en raison de la plus grande fréquence d'agneaux mort-nés en 3 en 2 (avortements dus à Toxoplasma Gondii). La mortalité des agneaux après l'âge de 10 j n'a pas été significativement différente entre les deux systèmes (4,2 vs 3,1 pour $3 e n 2$ et $1 /$ an respectivement). Les arthrites, les entérotoxémies et les pneumonies ont été les principales causes de mort, sans différences d'occurrence entre les deux systèmes.

\begin{tabular}{|c|c|c|c|c|c|c|c|c|c|}
\hline Mode d'engraissement & \multicolumn{3}{|c|}{ Au pâturage } & \multicolumn{2}{|c|}{ En bergerie } & \multirow[b]{2}{*}{ SEM } & \multicolumn{3}{|c|}{$P$} \\
\hline Système (S) & $1 / a n^{1}$ & $3 e n 2^{1}$ & $3 e n 2^{1}$ & $1 / a n^{1}$ & $3 e n 2^{1}$ & & $S$ & PMB & $\begin{array}{l}\mathrm{Sx} \\
\mathrm{PMB}\end{array}$ \\
\hline Période de mise bas (PMB) & Mars & Mars & Juin & Nov & Nov & & & & \\
\hline Nombre d'agneaux & 186 & 169 & 113 & 183 & 105 & & & & \\
\hline $\begin{array}{l}\text { Poids à la naissance }(\mathrm{kg}) \\
\text { Vitesse de croissance }(\mathrm{g} / \mathrm{j})\end{array}$ & 3,7 & 3,7 & 4,1 & 4,1 & 4,3 & 0,65 & NS & $<0,001$ & $<0,05$ \\
\hline $0-70 \mathrm{j}$ & 248 & 234 & 204 & 263 & 243 & 47,6 & $<0,001$ & $<0,001$ & NS \\
\hline $70 \mathrm{j}$ - abattage & 185 & 201 & 169 & 235 & 212 & 50 & NS & $<0,001$ & $<0,001$ \\
\hline $\begin{array}{l}\text { Age au sevrage }(\mathrm{j}) \\
\text { Poids au sevrage }(\mathrm{kg})\end{array}$ & $\begin{array}{c}89 \\
24,4\end{array}$ & $\begin{array}{c}58 \\
18,4\end{array}$ & $\begin{array}{c}69 \\
18,3\end{array}$ & $\begin{array}{c}74 \\
23,4\end{array}$ & $\begin{array}{c}69 \\
21,1\end{array}$ & $\begin{array}{l}13 \\
4,5\end{array}$ & $<0,001$ & $<0,01$ & 0,001 \\
\hline Age à l'abattage (j) & 146 & 149 & 154 & 122 & 125 & 27,8 & NS & $<0,001$ & NS \\
\hline Poids à l'abattage (kg) & 34,5 & 35,3 & 32,3 & 33,5 & 32,3 & 3,7 & NS & $<0,001$ & $<0,01$ \\
\hline
\end{tabular}

Tableau 2. Performances des agneaux vendus, selon le mode d'engraissement, le système d'élevage et la période de naissance.

1 1/an et 3en2 : un agnelage par brebis et par an et 3 agnelages en 2 ans, respectivement. 
Tableau 3. Caractéristiques et qualité des carcasses.

\begin{tabular}{|c|c|c|c|c|}
\hline Système & $1 / a n^{1}$ & $3 e n 2^{1}$ & SEM & $P$ \\
\hline Nombre d'agneaux & 356 & 396 & & \\
\hline Poids de la carcasse $(\mathrm{kg})$ & 15,52 & 15,08 & 2,19 & $<0,05$ \\
\hline Conformation (sur une échelle de 1 à 15) & 7,0 & 6,9 & 2,09 & NS \\
\hline Poids du gras périrénal $(\mathrm{g})$ & 230 & 220 & 100 & NS \\
\hline Couleur du gras dorsal & & & & \\
\hline Luminosité (L*) & 73,81 & 73,86 & 4,15 & NS \\
\hline Indice de rouge $\left(a^{*}\right)$ & 2,36 & 2,26 & 1,99 & NS \\
\hline Indice de jaune $\left(b^{*}\right)$ & 14,57 & 14,64 & 2,76 & NS \\
\hline Fermeté du gras dorsal (échelle de 1 à 15) & 11,1 & 10,2 & 2,31 & $<0,001$ \\
\hline
\end{tabular}

1 1/an et $3 e n 2$ : un agnelage par brebis et par an et 3 agnelages en 2 ans, respectivement. b) Parasites relevés lors des autop-

Les proportions des espèces de strongles digestifs n'ont pas varié significativement avant et après la conversion en $\mathrm{AB}$, avec toutefois une tendance à l'augmentation de la proportion de $T$. circumcincta. Il n'y a pas eu de différences significatives entre les deux systèmes, et les proportions observées sont proches de celles rencontrées dans des fermes $\mathrm{AB}$ de la région (Cabaret et al 2002). Le cestode Moniezia a été également présent dans les deux systèmes. L'insecte Oestrus ovis et le nématode protostrongle Muellerius capillaris étaient présents, mais avec une très faible intensité.

c) Niveau d'infestation parasitaire (tableau 6)

L'excrétion des œufs de strongles a été plus forte en 3en2 qu'en 1/an, aussi bien sies (tableau 5) pour les agneaux que pour les brebis $(\mathrm{P}<0,05)$. Il en était de même pour l'excrétion d'oocystes de coccidies chez les agneaux $(\mathrm{P}<0,05)$. Il n'y a pas eu de différence entre les deux systèmes en ce qui concerne Moniezia.

\section{d) Traitements anthelminthiques}

Nous avons utilisé le lévamisole ou le tartrate de pyrantel car les strongles du site y étaient sensibles (Berrag et al 2001), et ce site était indemne de douves et quasiment indemne de Oestrus ovis et Muellerius capillaris. Les agneaux ont été traités contre Moniezia par un produit commercial phytothérapique, mais son efficacité a été très réduite et on peut considérer qu'il n'y a pas eu de traitement contre ce parasite (Cabaret et al 2005). Très peu d'agneaux ont été exclus de la certification $\mathrm{AB}$ en raison d'un excès de traitements par des molécules de synthèse. Ainsi, en 2002, seulement 0,5\% (1/an)
Tableau 4. Principaux évènements pathologiques concernant les brebis mortes ou réformées.

\begin{tabular}{|l|c|c|c|}
\hline Evènements pathologiques & 1/an & $\mathbf{3 e n}^{1}$ & $\boldsymbol{P}$ \\
\hline Nombre de brebis & 112 & 112 & \\
Ensemble des évènements pathologiques & 18,5 & 28,6 & NS \\
(\% de brebis mortes ou réformées) & 10,8 & 15,6 & NS \\
Mammites (\% des brebis mortes ou réformées) & 7,1 & 9,4 & NS \\
Problèmes digestifs (\% des brebis mortes ou réformées) & 18,9 & 12,5 & NS \\
Maladies métaboliques (\% des pathologies identifiées) & 3,2 & 5,8 & NS \\
Problèmes de reproduction (\% des brebis réformées) & \multicolumn{2}{|l}{}
\end{tabular}

1 1/an et 3en 2 : un agnelage par brebis et par an et 3 agnelages en 2 ans, respectivement. et $0,7 \%$ (3en 2$)$ des agneaux produits ont été exclus de la certification $\mathrm{AB}$ pour cette raison.

\section{5 / Résultats économiques (tableau 7)}

Les résultats portent sur les années 2001 à 2003 ; pour 2001, seuls les agneaux nés en novembre ont été valorisés en $\mathrm{AB}$ (vente début 2002, labellisation $\mathrm{AB}$ acquise à cette date).

\section{a) Produit brut par brebis}

En moyenne sur la période 20012003 , le produit brut par brebis a été supérieur de $6 \%$ en 3 en 2 comparé à 1/an (140 vs $131 €)$. Il a par ailleurs été beaucoup plus variable en 3 en 2 , variant de $121 €$ à $170 €$, contre $120 €$ à $146 €$ en 1/an. L'élément déterminant du produit brut par brebis a été le nombre d'agneaux vendus, dépendant directement de la productivité numérique par brebis, car $i$ ) le prix de vente a été globalement comparable entre les 2 systèmes $(5,22 €$ par $\mathrm{kg}$ de carcasse en 3 en 2 et $5,24 €$ en $1 / a n$ ), et ii) le poids de carcasse n'a été que légèrement inférieur en 3en2. En 2002, année où la productivité numérique par brebis a été la plus élevée en $3 e n 2$, le produit brut par brebis a été supérieur de $24 €$ en 3 en 2 ( 170 vs $146 €$ en $1 / a n)$. De 2001 à 2003, le nombre d'agneaux vendus par quinzaine a été en moyenne de 6,6 (écart type de 6,0) pour 1/an et de 6,8 en $3 e n 2$ (écart type de 3,5 ). La moindre variabilité en 3 en 2 indique que ce système a assuré une plus grande régularité de mise en marché, avec des agneaux vendus au quatrième trimestre, lorsque l'offre en agneaux est souvent réduite (figure 6). bis

b) Charges opérationnelles par bre-

En moyenne sur la période 20012003, les charges opérationnelles par brebis ont été supérieures de $22 \%$ en 3en2, comparativement à $1 /$ an $(81$ vs $66 €)$, en lien avec une plus forte utilisation de concentrés (achetés ou pro-

Tableau 5. Proportions des principaux strongles digestifs avant et après la conversion en $A B$.

\begin{tabular}{|l|c|c|c|c|c|c|}
\hline & \multicolumn{2}{|c|}{ Avant conversion } & \multicolumn{2}{c|}{ 1/an $^{1}$} & \multicolumn{2}{c|}{ 3en2 $^{1}$} \\
\cline { 2 - 7 } Teladorsagia circumcincta & Brebis & Agneaux & Brebis & Agneaux & Brebis & Agneaux \\
\cline { 2 - 7 } Haemonchus contortus & 0 & 31 & 30 & 15 & 18 & 81 \\
Trichostrongylus axei & 0 & 7 & 1 & 2 & 1 & 2 \\
Trichostrongylus colubriformis & 44 & 31 & 30 & 29 & 53 & 4 \\
Cooperia curticei & 40 & 15 & 11 & 2 & 16 & 2 \\
\hline
\end{tabular}

1 1/an et 3en2 : un agnelage par brebis et par an et 3 agnelages en 2 ans, respectivement. 
Tableau 6. Excrétion d'oeufs de strongles, de Moniezia et d'oocystes de coccidies (valeur moyenne et étendue).

\begin{tabular}{|l|l|c|c|c|}
\hline \multicolumn{1}{|c|}{ Parasites } & Animaux & 1/an & 3en2 & $P$ \\
\hline Strongles digestifs & Brebis & $325(10-800)$ & $465(10-1050)$ & $<0,05$ \\
Strongles digestifs & Agneaux & $885(10-2000)$ & $1322(10-2750)$ & $<0,05$ \\
Coccidia & Agneaux & $42114(0-130000)$ & $86426(100-190000)$ & $<0,05$ \\
Moniezia (\%) & Agneaux & $16(5-35)$ & $23(0-55)$ & NS \\
\hline
\end{tabular}

11/an et 3en 2 : un agnelage par brebis et par an et 3 agnelages en 2 ans, respectivement.

2 Pour Moniezia, il s'agit de la proportion moyenne (et de son étendue) des agneaux émettant des oeufs dans leurs fèces.

Tableau 7. Résultats économiques : moyenne (écart type) sur la période 2001-2003.

\begin{tabular}{|l|c|c|}
\hline & $1 /$ an $^{1}$ & 3 en2 \\
\cline { 2 - 3 } Concentré/brebis $(€)$ & $39,3(6,7)$ & $49,5(9,2)$ \\
Concentré/brebis $(\mathrm{kg})$ & $121(12,0)$ & $156(21,2)$ \\
Fourrage acheté/brebis $(€)$ & $9,0(10,3)$ & $10,3(14,3)$ \\
Prix de vente des agneaux & $5,22(0,42)$ & $5,24(0,31)$ \\
$(€ /$ kg carcasse) & $131(13,4)$ & $140(26,6)$ \\
Produit brut/brebis $(€)$ & $66(11,1)$ & $81(10,1)$ \\
Charges opérationnelles/brebis $(€)$ & $65(18,8)$ & $59(29,8)$ \\
Marge Brute/brebis $(€)$ & $73,2(8,3)$ & $68,6(11,3)$ \\
Autonomie fourragère $(\%)$ & $78,7(7,8)$ & $75,6(9,3)$ \\
Autonomie alimentaire $(\%)$ &
\end{tabular}

11/an et 3en2 : un agnelage par brebis et par an et 3 agnelages en 2 ans, respectivement.

duits ; 49,5 € en 3en 2 vs 39,3 € en 1/an). Ce surcoût de concentrés dans le système 3 en 2 provient principalement de $i)$ l'augmentation du rythme d'agnelage qui a conduit à une utilisation plus importante de concentré dans l'alimentation des brebis, ii) l'augmentation du nombre d'agneaux produits par brebis, et iii) la diminution de l'âge des agneaux au sevrage. Les autres coûts de production (frais vétérinaires et frais divers d'élevage) ont été supérieurs de $19 \%$ en 3 en 2 (16,3 vs 13,7 €/ brebis en $1 / a n)$. Les frais vétérinaires par brebis en particulier (honoraires et produits vétérinaires) ont représenté 5,6 $€$ en 3en 2 vs $4 €$ en 1/an. Au cours de la période de conversion (2000-2001), les frais vétérinaires par brebis ont été de trois à quatre fois plus élevés qu'après la conversion, principalement en raison de l'adoption de mesures de prévention contre les boiteries $(7,4 €$ et $10 €$ en $1 / a n$ et $3 e n 2$ pendant la conversion, puis $2,2 €$ et $2,5 €)$.

Pour l'année 2002, l'écart de charges opérationnelles entre les deux systèmes a atteint 34\% (80 € en 3en 2 vs $60 €$ en $1 / a n$ ), toujours en lien avec le niveau d'utilisation d'aliments concentrés, dont la consommation totale a atteint $178 \mathrm{~kg}$ par brebis pour 3 en2 $(57,5 € € /$ brebis) et $113 \mathrm{~kg}$ en $1 /$ an $(36,3 € /$ brebis). Le déficit fourrager lié à une courte sécheresse en 2002 a nécessité l'achat de foin à hauteur de $7 €$ par brebis pour $1 /$ an et $4 €$ pour $3 e n 2$ (respectivement 46 et $28 \mathrm{~kg}$ par brebis). En 2003, la sécheresse beaucoup plus marquée a conduit à des achats de foin à hauteur de $20 €$ par brebis pour 1/an et $27 €$ en 3 en 2 , soit respectivement 150 et $197 \mathrm{~kg}$ par brebis, ce qui a représenté près de 50 et $60 \%$ des besoins hivernaux.

\section{c) Marge brute par brebis}

En moyenne sur la période 20012003, la marge brute par brebis a été plus élevée pour 1/an que pour 3 en 2 (65 vs $59 €$, respectivement). Elle a également été moins fluctuante pour 1/an, variant de 49 à $86 €$ contre 31 à $90 €$ pour 3 en2. Même en 2002, lorsque la productivité numérique par brebis a été excellente en $3 e n 2$, la marge brute par brebis a été peu différente entre les deux systèmes $(90 €$ en 3 en 2 vs $86 €$ en 1/an) en raison des quantités d'aliments concentrés. En 2003, les conditions de sécheresse ont fortement pénalisé la marge brute par brebis dans les deux systèmes, en raison de l'augmentation des achats de fourrages ; cette année là, le système 3 en 2 a par ailleurs subi une forte baisse de la fertilité lors de la lutte de fin de printemps. La marge brute par brebis est alors tombée à $31 €$ en 3 en 2 et $49 €$ en $1 / a n$.

\section{3 / Discussion}

\section{1 / Eléments de réflexion sur la conduite de deux troupeaux ovins en agriculture biologique}

a) Consommation de fourrage, autonomies fourragère et alimentaire et bilans minéraux

Les consommations annuelles de fourrage (foin et herbe pâturée) ont

Figure 6. Ventes d'agneaux par quinzaine (moyenne 2001-2003), pour les systèmes 1/an et 3en2.

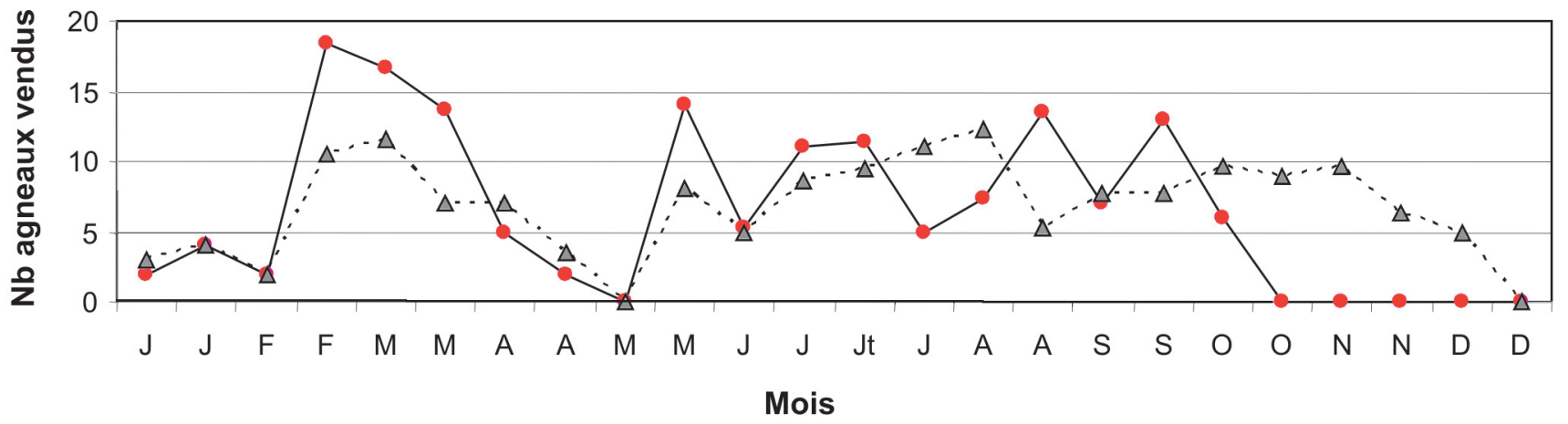


atteint 320 et $526 \mathrm{~kg}$ MS par brebis (les brebis ont pâturé en moyenne pendant $223 \mathrm{j}$ par an). Une expérimentation parallèle (Tournadre et al 2006) a montré qu'une limitation du concentré à $40 \%$ de la ration conduisait à une forte augmentation de la quantité de foin ingérée par les agneaux en bergerie : $25 \mathrm{~kg}$ MS/agneau contre $6 \mathrm{~kg}$ MS/agneau lorsque le concentré est distribué ad libitum et qu'il représente alors $80 \%$ de la MS de la ration. L'utilisation de cette référence dans le système 1/an conduit à évaluer la part des fourrages grossiers récoltés consommés par les agneaux à 9\% des besoins totaux en fourrages stockés, contre $2 \%$ si les agneaux étaient engraissés avec une distribution ad libitum du concentré, comme cela est pratiqué en élevage conventionnel dans la même région (Laignel et Benoit 2004). La consommation importante de fourrages (stockés ou pâturés) par les agneaux a contribué, malgré des disponibilités fourragères restreintes pour deux années sur trois (2002 et 2003, figure 7), à l'obtention d'une autonomie fourragère de $73,2 \%$ en $1 /$ an et de $68,6 \%$ en 3 en 2 (tableau7).

La recherche d'une autonomie alimentaire élevée $(78,7 \%$ en $1 / a n$ vs $75,6 \%$ en $3 e n 2$, avec des conditions climatiques difficiles) est passée par une utilisation importante de fourrages, mais aussi par la culture de céréales, qui ont fourni du tiers à la moitié des besoins en concentrés, et qui ont permis une forte réduction des achats d'aliments par rapport à la précédente expérimentation sur ce site (Thériez et al 1997a). Par ailleurs, aucun engrais exogène n'a été utilisé, la seule fertilisation ayant consisté en l'épandage du compost issu du fumier de la ferme ; aussi, la seule entrée de minéraux sur les systèmes expérimentés a été réalisée par les aliments achetés (et la paille), à hauteur de $15,5 \mathrm{~kg}$ de $\mathrm{N} / \mathrm{ha} /$ an et de $2,7 \mathrm{~kg} \mathrm{P} / \mathrm{ha} / \mathrm{an}$ en moyenne pour les deux systèmes. On pourrait ainsi craindre, à terme, des déficits minéraux. Le souci de maintenir la fertilité des sols sur le long terme (Mogensen et al 2007) justifie ainsi l'utilisation d'outils de suivi de la fertilité (utilisation des indices de nutrition), et nécessite de réfléchir à la meilleure façon d'utiliser les engrais de ferme (fumier/compost) pour une valorisation optimale de cette ressource. Cependant, dans notre situation, l'exportation de minéraux par le biais de la viande vendue a été faible : à l'échelle globale des systèmes et en moyenne sur la durée de l'expérimentation, elle a été seulement de $5,5 \mathrm{~kg} \mathrm{~N} / \mathrm{ha} / \mathrm{an}$ et $2,3 \mathrm{~kg}$
$\mathrm{P} /$ ha/an via la production de viande (en moyenne $235 \mathrm{~kg}$ poids vif/ha/an). L'azote et le phosphore introduits dans les systèmes via les achats d'aliments représentent ainsi respectivement trois fois et une fois les sorties sous forme de viande vendue. L'indice de nutrition azotée des fourrages était de niveau moyen en 2000 (69), mais faible en 2002 (57), reflet direct de la diminution des légumineuses. Les indices de nutrition $\mathrm{P}$ et $\mathrm{K}$ sont restés corrects : 112 et 102 pour $\mathrm{P}, 106$ et 99 pour K, respectivement en 2000 et 2002. Ainsi, une bonne gestion des matières organiques a semblé suffisante pour assurer le maintien d'une fertilité correcte du sol en $\mathrm{P}$ et $\mathrm{K}$; quant à la fertilité azotée, elle est pour partie assurée par la présence de légumineuses dans les prairies et dépend donc de leur abondance. Les pertes d'azote par lessivage sont minimes à l'échelle globale des systèmes compte tenu de la part importante des surfaces fourragères (pacages et prairies permanentes) et du type de fertilisation (compost). Notons cependant qu'au-delà de la vision du bilan minéral global à l'échelle du système, l'utilisation des effluents d'élevage doit être réfléchie en fonction du type d'utilisation de chaque parcelle (pâturage, fauche, rotation de cultures...).

\section{b) Résultats zootechniques}

Les surfaces du site support de l'étude avaient été conduites extensive-

Figure 7. Autonomies fourragère et alimentaire (UF), pour les systèmes 1/an et 3en2.

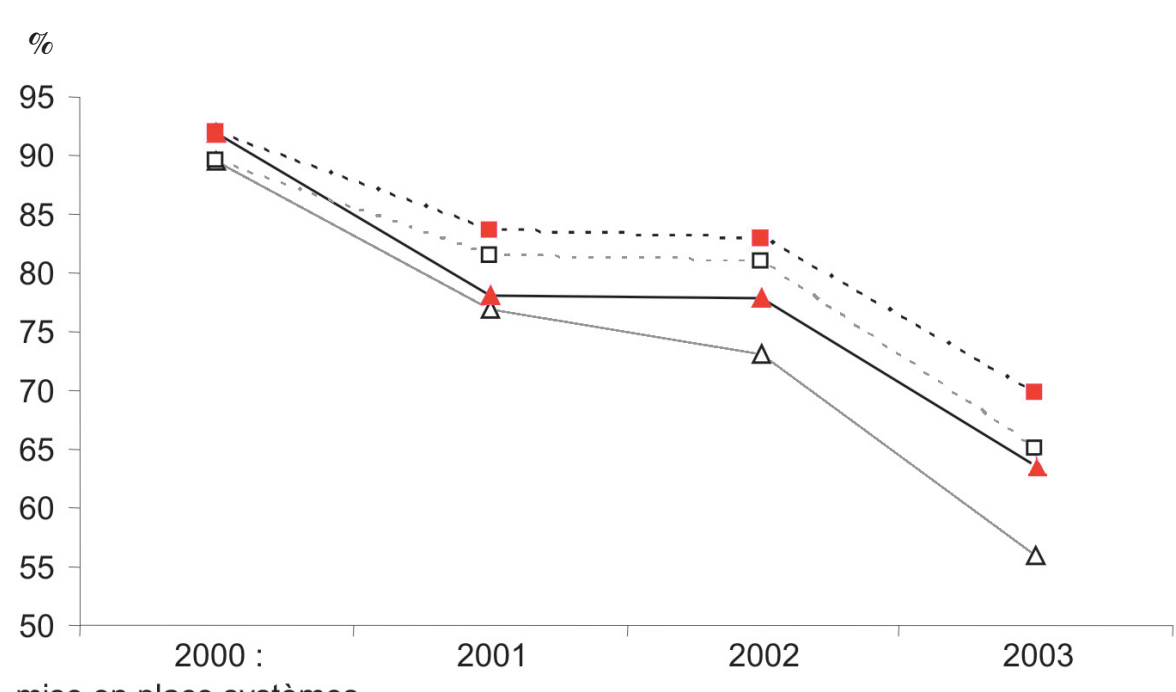

mise en place systèmes ment durant les 10 années précédant cette expérimentation (Thériez et al 1997a). La conversion à l'agriculture biologique n'a pas été un changement radical; le système 1/an a présenté une productivité numérique en retrait de $8,8 \%$ par rapport à celle obtenue précédemment sur ce troupeau (conduite extensive des surfaces) avec l'utilisation de traitements hormonaux et la même race. Elle atteint ainsi 1,45 agneau vivant par brebis pouvant mettre bas en 1/an vs 1,60 pour les études précédentes (Thériez et al 1997a). Le dénominateur retenu dans ce calcul de la productivité numérique (brebis pouvant mettre bas) est adapté au mode de conduite différent des agnelles entre les deux expérimentations (Marzin et al 1984). La baisse de productivité numérique par brebis est essentiellement liée à celle de la prolificité (166\% contre $181 \%$ ), alors que la fertilité est meilleure $(93,4$ vs $81,5 \%)$. Le non-usage de traitement hormonal n'a donc pas pénalisé la fertilité mais seulement la prolificité. Nous avons fait le choix de la même race de brebis pour les deux systèmes expérimentés afin de faciliter la comparaison entre eux. Toutefois, il aurait pu être envisagé d'utiliser deux races différentes, chacune adaptée aux objectifs spécifiques de chaque système (Nauta et al 2008). En particulier, il pourrait être pertinent de choisir une race ayant un meilleur potentiel de reproduction à

\section{Années \\ $\Delta-$ 3en 2 : autonomie fourragère $\longrightarrow-1 /$ an : autonomie fourragère \\ - - - - 3en2 : autonomie alimentaire …- - 1/an : autonomie alimentaire}


contre saison, afin d'assurer une fertilité plus régulière.

\section{c) Gestion de la santé}

La stratégie de prévention sanitaire mise en œuvre a permis un contrôle satisfaisant sur une période de 4 ans à dater du début de la conversion en AB. Le nombre d'animaux exclus de la certification $\mathrm{AB}$ en raison du nombre de traitements allopathiques chimiques a été très faible. Les mortalités, tant des brebis que des agneaux, sont restées dans les deux systèmes à un niveau habituel, si l'on se réfère aux valeurs antérieures à la conversion. Au-delà de l'AB, la réduction du nombre de traitements anthelminthiques de synthèse présente l'intérêt très général de limiter la pression de sélection favorable à l'acquisition de résistances des strongles à ces produits. La gestion du parasitisme a été menée sur les mêmes principes pour les deux systèmes expérimentés ; ils méritent d'être explicités, car ils peuvent parfois s'écarter de deux principes en $\mathrm{AB}$, la préférence pour les méthodes de traitement alternatives et le non recours aux traitements préventifs. Les méthodes alternatives ne sont pas encore suffisamment validées dans la sphère parasitaire (Hoste et al 2009). De plus, pour des raisons éthiques (Hermansen 2003), nous avons fait le choix d'utiliser, de façon économe, des traitements allopathiques de synthèse actifs sur les strongles, pour les agneaux ayant des performances médiocres, et en particulier aux périodes de fortes infestations parasitaires. Cette stratégie repose donc sur un suivi attentif des animaux, qui peut également s'adosser à des analyses de laboratoire pour évaluer l'infestation. De plus, nous avons traité les brebis contre les strongles avant la sortie à l'herbe, sachant que c'est un des points clés qui peut déterminer la contamination future des agneaux (Athanasiadou et al 2007). Un raffinement de la méthode serait de ne traiter que les brebis proches de la date de mise bas ou ayant agnelé dans le mois précédant la mise à l'herbe, car c'est durant cette période que les brebis excrètent un nombre accru d'œufs de strongles dans leurs fèces, source de contamination future des agneaux. C'est d'ailleurs la différence d'excrétion durant cette période proche de la mise bas qui explique la plus grande excrétion d'œufs de strongles par les brebis et les agneaux dans le troupeau 3en2.

\section{2 / Comparaison des deux sys- tèmes d'élevage}

a) Production animale et qualité des agneaux

Malgré une grande différence entre les deux systèmes dans l'organisation de la reproduction des animaux, la productivité numérique par brebis a été inférieure de seulement $7 \%$ dans le système 1/an par rapport au système 3 en 2 . En outre, la productivité numérique par brebis a été beaucoup plus variable en $3 e n 2$, reflet essentiellement de la fertilité qui a atteint des niveaux parfois très faibles pour les mises bas de novembre et de juin. Le faible avantage de productivité numérique par brebis pour le système 3 en 2 est dû principalement à $i$ ) la forte sollicitation des brebis dans ce système de conduite, avec un impact négatif sur leur fertilité, en particulier à la lutte de juin, ii) un moins bon état sanitaire des animaux, avec une mortalité des agneaux et un niveau parasitaire plus élevés en 3 en2. De plus, la qualité des agneaux produits a été moindre en 3 en2, avec un poids moyen de la carcasse plus faible et une fermeté du gras de couverture un peu inférieure, en raison sans doute d'un âge inférieur au sevrage et d'une part de concentré plus importante dans la ration (Thériez et al 1997b, Priolo et al 2002).

\section{b) Conduite des troupeaux, travail et} économie

Le troupeau du système $3 e n 2$ a été plus difficile à gérer en raison du plus grand nombre de lots d'animaux et de la concurrence entre lots pour les fourrages de bonne qualité. Le travail de l'éleveur est en effet augmenté et rendu plus complexe lorsque le nombre de lots augmente (Cournut et Dedieu 2004). Dans le système 3en2, la période la plus difficile a été le mois de juin, avec une forte concurrence pour les pâturages de bonne qualité entre les agneaux, les brebis allaitantes et les brebis en lutte. En outre, le niveau parasitaire des brebis mettant bas en juin a été élevé, en relation avec l'excrétion accrue d'œufs de strongles des brebis à ce stade physiologique.

Malgré une meilleure répartition des ventes des agneaux dans le système 3en 2, la marge brute observée dans ce système a été plus faible en moyenne et plus variable que celle du système $1 / a n$, principalement en raison des charges d'alimentation plus élevées. Dans un contexte économique de forte augmentation du prix des principaux intrants (concentrés, céréales et énergie), ce qui a été le cas en 2008, l'avantage économique du système 1/an pourrait être encore accru du fait de sa meilleure autonomie alimentaire, propriété qui lui confère par ailleurs une meilleure efficacité énergétique globale (Benoit et Laignel 2008).

\section{c) Limites du système 3 en 2 en $A B$}

Dans la conjoncture des années 2001 à 2003, le système $3 e n 2$ n'a pas dégagé une meilleure marge brute que le système 1/an. Un prix de vente des agneaux supérieur lui donnerait un certain intérêt, mais le contexte 2008 montre plutôt un renchérissement des coûts de production, situation qui lui est défavorable. La mise en œuvre de ce système et son pilotage se sont montrés plus complexes, sans plus-value économique. En effet, ce système subit plus de contraintes, et en premier lieu pour ce qui concerne les périodes de lutte possibles : afin d'éviter la période d'anoestrus de printemps, il a été nécessaire de fixer une session de lutte en juin qui conduit à une lutte ultérieure, pour ces mêmes brebis, en janvier. Compte tenu du niveau de sollicitation des brebis, non seulement les résultats de reproduction ont été irréguliers, mais surtout, la mise bas de juin a généré de nombreuses contraintes qui ont été soulignées (concurrence pour des ressources fourragères de bonne qualité, impact du parasitisme). Le choix d'une race moins saisonnée permettrait de laisser plus de latitude sur le choix de dates de reproduction, mais d'autres limites pourraient alors apparaître (conformation des agneaux, en particulier). Les «degrés de liberté» apparaissent ainsi moins nombreux en $3 e n 2$ qu'en 1/an: peu de latitude de choix de dates de mise bas, peu de phases de récupération possibles pour les brebis (état corporel), et dépendance pour les ressources fourragères de bonne qualité à des périodes clés. Le cahier des charges et surtout le coût des intrants (aliments concentrés) limitent les possibilités d'intervention et les marges de manoeuvre. Les conditions relativement difficiles du milieu (sol) et les conditions climatiques assez sévères ont révélé cette plus grande difficulté d'adaptation du système $3 e n 2$, avec les conséquences technico-économiques correspondantes. Ce système aurait probablement des atouts à extérioriser dans un contexte pédoclimatique plus favorable permettant en particulier une période de pâturage plus longue et plus assurée. La tension exercée au travers du rythme de reproduction se traduit in fine par une plus grande variabilité des 
résultats zootechniques et économiques.

\section{3 / Comparaison des deux sys- tèmes à un groupe de fermes privées (année 2002, tableau 8)}

Les résultats technico-économiques des deux systèmes étudiés ont été comparés à ceux obtenus en 2002 dans 10 fermes en $\mathrm{AB}$ et 16 fermes conventionnelles (Laignel et Benoit 2004). Cette comparaison est indicative, car les conditions d'élevages sont parfois assez différentes, notamment au niveau des races, de la gestion de la santé et du travail, et de la qualité des terres cultivées et des pâturages.

La consommation moyenne d'aliments concentrés a été de $133 \mathrm{~kg}$ et 154 $\mathrm{kg}$ par brebis respectivement dans les fermes $\mathrm{AB}$ et conventionnelles, contre $113 \mathrm{~kg}$ par brebis en $1 / \mathrm{an}$ et $178 \mathrm{~kg}$ en 3en2. La faible consommation de concentré dans le système 1/an est liée à l'importance de la part de l'herbe pâturée dans l'alimentation des animaux, qui concourt à un niveau élevé d'autonomie fourragère et assure le lien au sol recommandé par le cahier des charges $\mathrm{AB}$. Le niveau de consommation des aliments concentrés est d'une importance primordiale en $\mathrm{AB}$, tant pour l'autonomie alimentaire que pour le coût $(0,32 € / \mathrm{kg}$ d'aliment concentré pour les deux systèmes étudiés contre $0,20 € / \mathrm{kg}$ pour les exploitations conventionnelles, soit un surcoût de $60 \%$ des concentrés $\mathrm{AB}$ ).

Dans la présente étude, les dépenses de santé par brebis sont restées faibles (2,5 $€$ en 2002-2003 contre $4 €$ dans les fermes privées $\mathrm{AB}$ ou conventionnelles), et du niveau de celles observés dans les fermes $\mathrm{AB}$ obtenant de bons résultats technico-économiques. Les dépenses de santé sont le fruit de stratégies des éleveurs qui se fondent sur des aspects techniques mais surtout sur des conceptions individuelles basées sur des valeurs (Cabaret et Nicourt 2009). Il apparaît que le recours aux médecines alternatives peut s'ajouter au recours aux thérapeutiques classiques chez certains éleveurs, les dépenses de santé étant alors parfois particulièrement augmentées. Le fait de travailler en conditions expérimentales (ce qui a permis de réaliser des diagnostics réguliers) et les choix thérapeutiques que nous avons faits (utilisation parcimonieuse et parfois préventive de molécules chimiques de synthèse) ont permis de maintenir ces coûts tout en maîtrisant la santé des animaux.

Le poids moyen de carcasse des agneaux issus des fermes privées en $\mathrm{AB}$ a été supérieur $(16,2$ vs $15,1 \mathrm{~kg}$ et 14,7 $\mathrm{kg}$ en $1 / \mathrm{an}$ et $3 e n 2$ respectivement) et $70 \%$ des agneaux ont été vendus sur le marché $\mathrm{AB}$, comparativement à $67 \%$ en $1 / a n$ et $64 \%$ en $3 e n 2$. Le poids moyen de carcasse des agneaux des fermes conventionnelles a également été supérieur $(16,9 \mathrm{~kg})$, mais ces agneaux ont été vendus à un prix inférieur, à 4,90 $€ / \mathrm{kg}$ contre $5,53 € / \mathrm{kg}$ dans les fermes $\mathrm{AB}$ et $5,30 € / \mathrm{kg}$ dans la présente étude.

La productivité numérique par brebis a été plus élevée que celle observée en moyenne dans les fermes privées, qu'elles soient $\mathrm{AB}$ ou conventionnelles, avec $152 \%$ en $1 /$ an et $193 \%$ en $3 e n 2$ contre $135 \%$ en moyenne dans les fermes privées. La productivité numérique par brebis dans le système $3 e n 2$ a été en moyenne proche de celle observée dans les 6 fermes conventionnelles pratiquant le même rythme de reproduction (163\% en moyenne en 2002). En 2002, les marges brutes observées dans la présente étude ( $86 €$ par brebis en $1 /$ an

Tableau 8. Comparaison des résultats économiques des systèmes étudiés avec ceux de fermes privées en AB ou en conventionnel (année 2002).

\begin{tabular}{|c|c|c|c|c|}
\hline & $1 / a n^{1}$ & $3 e n 2^{1}$ & $\begin{array}{c}\mathrm{AB} \\
n=10\end{array}$ & $\begin{array}{c}\text { Conventionnel } \\
n=16\end{array}$ \\
\hline Concentré/brebis $(\mathrm{kg})$ & 113 & 178 & 133 & 154 \\
\hline $\begin{array}{l}\text { Prix de vente des agneaux } \\
\text { ( } € / \mathrm{kg} \text { carcasse) }\end{array}$ & 5,30 & 5,29 & 5,53 & 4,90 \\
\hline Produit brut/brebis $(€)$ & 146 & 170 & 131 & 140 \\
\hline Charges opérationnelles/brebis $(€)$ & 60 & 80 & 66 & 55 \\
\hline Marge Brute/brebis $(€)$ & 86 & 90 & 65 & 85 \\
\hline Autonomie fourragère $(\%)$ & 77,9 & 73,2 & - & 72,2 \\
\hline Autonomie alimentaire $(\%)$ & 82,9 & 80,6 & - & 77,1 \\
\hline
\end{tabular}

11/an et 3en 2 : un agnelage par brebis et par an et 3 agnelages en 2 ans, respectivement. et $90 €$ en 3 en 2 ) ont été comparables à celles des fermes privées conventionnelles ( $85 €$ par brebis), et nettement plus élevées que celle observées dans les fermes $\mathrm{AB}(65 €$ par brebis en moyenne) qui affichent à la fois des productivités numériques plus faibles et des coûts de production plus élevés. Ce dispositif en site expérimental s'est ainsi montré plus efficace du point de vue économique. En particulier, la stratégie adoptée dans le système $1 /$ an avec un plafonnement de la productivité numérique (non accélération de la reproduction) et une économie d'intrants importante, s'est révélée particulièrement intéressante. Cette recherche de cohérence de systèmes d'élevage en fonction des conditions locales de production peut être identifiée dans d'autres dispositifs en $\mathrm{AB}$ (Benoit et Laignel 2009).

\section{Conclusion}

Cette étude montre que, contrairement à ce qui est observé en élevage conventionnel, l'accélération du rythme de reproduction en élevage ovin biologique n'a pas généré dans notre étude d'amélioration des résultats économiques par rapport à un système plus classique ne comportant qu'un seul agnelage par an et par brebis. Ce système $3 e n 2$, risqué en $\mathrm{AB}$, ne pouvait être évalué que dans le cadre d'une ferme expérimentale. Les conditions de milieu relativement difficiles du site expérimental et les conditions climatiques des trois années étudiées ont certainement exacerbé le contraste observé entre les deux systèmes d'élevage expérimentés et clairement montré les limites du système $3 e n 2$. Au final, ses résultats technico-économiques sont plus aléatoires (variabilité entre années) et sa gestion est plus difficile que celle d'un système moins intensif de reproduction. Le système avec un seul agnelage par brebis et par an est resté à la fois très efficace du point de vue de l'animal, avec une bonne productivité des brebis, un meilleur contrôle du parasitisme, et une forte auto-suffisance pour l'alimentation du troupeau. Dans la conjoncture de l'année 2002 , les résultats technico-économiques de ce système ont été du même niveau que ceux d'exploitations conventionnelles relativement performantes de la même région ; cependant, étant conduit en $\mathrm{AB}$, ce système d'élevage est mieux positionné face à la demande sociale de produits «naturels» issus de systèmes de production préservant l'environnement. 


\section{Remerciements}

Cette étude a été financée grâce à l'aide du FEOGA Massif Central et du CIAB (INRA). Nous remercions particulièrement M. Petit, F. Bocquier, M.
Bouilhol, J. Barnouin, P. Jacquiet et H. Hoste pour leurs conseils précieux dans la conception de ce dispositif, et J.-Y. Pailleux, M. Verdier, R. Jailler, Y. Thomas, A. Guittard, J. Cortet, C. Sauvé et J-.P. Chacornac pour la conduite de l'expérimentation et la réalisation des mesures. Nous remercions également plusieurs étudiants pour leur collaboration : M.-F. Millord, P. Treissac, L. Simmoneau et V. Gonnord.

\section{Références}

Agence Bio, 2007. http://www.agencebio.org/ upload/pagesEdito/fichiers/production-BioFrance2007.pdf

Athanasiadou S., Gray D., Younie D. Tzamaloukas O., Jackson F., Kyriazakis I., 2007. The use of chicory for parasite control in organic ewes and their lambs. Parasitology, 134, 299307.

Aufrère J., Michalet-Doreau B., 1988 Comparison of methods for predicting digestibility of feeds. Anim. Feed Sci. Technol., 20, 203218.

Benoit M., 1998. Un outil de simulation du fonctionnement du troupeau ovin allaitant et de ses résultats économiques : une aide pour l'adaptation à des contextes nouveaux. INRA Prod. Anim., 11, 199-209.

Benoit M., Veysset P., 2003. Conversion of cattle and sheep suckler farming to organic farming: adaptation of the farming system and its economic consequences. Livest. Prod. Sci., 80, 141-152.

Benoit M., Laignel G., 2006. Méthodologie d'élaboration de résultats technico-économiques en élevage ovin allaitant. Illustration en France, en zone de plaine et de montagne. Options Méd., Série A, Séminaires Méditerranéens, 70, 57-65.

Benoit M., Laignel G., 2008. Sheep-for-meat farming systems in French semi-upland area. Adapting to new context: increased concentrates and energy prices, and new agricultural policy. $8^{\text {th }}$ Eur. IFSA Symp. Empowerment of the rural actors: a renewal of Farming Systems perspectives. 6-10 July, Clermont-Ferrand, France, 755764.

Benoit M, Laignel G., 2009. Performances techniques et économiques en élevage biologique d'ovins viande : observations en réseaux d'élevage et fermes expérimentale. $\mathrm{N}^{\circ}$ spécial Elevage bio. INRA Prod. Anim., 22, 197-206.

Benoit M., Laignel G., Liénard G., 1999. Facteurs techniques, cohérence de fonctionnement et rentabilité en élevage ovin allaitant. Exemples du Massif Central Nord et du Montmorillonnais. Renc. Rech. Rum., 6, 19-22.

Bentounsi B., Attir B., Meradi S., Cabaret J., 2007. Repeated treatment faecal egg counts to identify gastrointestinal nematode resistance in a context of low-level infection of sheep on farms in eastern Algeria. Vet. Parasitol., 144.

Berrag B., Silvestre A., Chartier C., Gasnier N., Laignel V., Tournadre H., Ballet J., Cabaret J., 2001. Low frequency of anthelminthic treatments does not prevent from nematode resistance in sheep and goats: four regional examples in field conditions. 18 th Int. Conf. World Ass. Adv. Vet. Parasitol., 26-30 August, Stresa, Italy, 120.

Brelurut A., 1987. Productivité d'un troupeau de brebis selon le rythme de reproduction. Bull. Tech. CRZV Theix, 68, 5-10.
Cabaret J., Nicourt C., 2009. Les problèmes sanitaires en élevage biologique : réalités, conceptions et pratiques. $\mathrm{N}^{\circ}$ spécial Elevage bio. INRA Prod. Anim., 22, 235-244.

Cabaret J., Dakkak A., Bahaida B., 1980. On some factors influencing the output of the larvae of prostrongylids of sheep in natural infections. Vet. Quart., 2, 115-120.

Cabaret J., Bouilhol M., Mage C., 2002. Managing helminths of ruminants in organic farming. Vet. Res., 33, 625-640.

Cabaret J., Gonnord V., Cortet J., Sauvé C., Ballet J., Tournadre H., 2005. Moniezia chez l'agneau d'herbe : épidémiologie et tentative de contrôle par un traitement alternatif. Alter Agri, 72, 4-7.

Cognié Y., Schirar A., Martinet J., Poulin N., Mirman B., 1984. Activité reproductrice et maitrise de l'ovulation chez la brebis. 9èmes Journ. Rech. Ovine Caprine, 109-133.

Cournut S., Dedieu B., 2004. A discrete events simulation of flock dynamics: a management application to three lambings in two years. Anim. Res., 53, 383-403.

Doyle C.J., Topp C.F.E., 2004. The economic opportunities for increasing the use of forage legumes in north European livestock systems under both conventional and organic management. Renewable Agric. Food Syst., 19, 15-22.

Fisher A.V., Enser M., Richardson R.I., Wood J.D., Nute G.R., Kurt E., Sinclair L.A., Wilkinson R.G., 2000. Fatty acid composition and eating quality of lamb types derived from four diverse bree x production systems. Meat Sci., 55, 141-147.

Hermansen J.E., 2003. Organic livestock production systems and appropriate development in relation to public expectations. Livest. Prod. Sci., 80, 3-15.

Hoste H., Cabaret J., Grosmond G., Guitard J.P., 2009. Alternatives aux traitements anthelminthiques en élevage biologique. $\mathrm{N}^{\circ}$ spécial Elevage bio. INRA Prod. Anim., 22, 245-254.

Hovi M., Sundrum A., Thamsborg S.M., 2003. Animal health and welfare in organic livestock production in Europe: current state and future challenges. Livest. Prod. Sci., 80, 41-43.

INRA Institut National de la Recherche Agronomique, 1989. Ruminant nutrition. Recommended allowances and feed Tables. Jarrige R (Ed). INRA Editions, Paris, France, $389 \mathrm{p}$

Laignel G., Benoit M., 2004. Production de viande ovine en agriculture biologique comparée à l'élevage conventionnel : résultats technicoéconomiques d'exploitations de plaine et de Montagne du Nord Massif Central. INRA Prod. Anim., 17, 133-143.

MAAF, 1986. Manual of veterinary parasitology laboratory techniques. Ministry of
Agriculture, Fisheries and Food. MAFF Reference Book 418, 159p.

Marzin J., Brelurut A., 1979. Performances comparées des brebis Limousines et croisés Romanov en conduite relativement intensives. Bull. Tech. CRVZ Theix, 37, 15-23.

Marzin J., Liénard G., 1984. Productivité en agneaux des troupeaux ovins. Réflexions sur le choix d'une méthode d'analyse. Bull. Tech. CRVZ Theix, 56, 69-90.

Mogensen L., Kristensen T., Danfaer A., 2007. Prototyping of organic dairy production systems self-sufficient with feed. Consequences on productivity, economy and nutrient balance. Biol. Agric. Horticult., 25, 13-35.

Nardone A., Zervas G., Ronchi B., 2004 Sustainability of small ruminant organic systems of production. Livest. Prod. Sci., 90, 27-39.

Nauta W.J., Baars T., Saatkamp H., Weenink D., Roep D., 2008. Farming strategies in organic dairy farming: Effects on breeding goal and choice of breed. An explorative study. Livest. Sci., 121, 187-199.

Prache S., Brelurut A., Thériez M., 1986. L'élevage de l'agneau à l'herbe. I. Effets de l'âge au sevrage sur les performances d'agneaux élevés à l'herbe puis engraissés en bergerie. Ann. Zootech., 35, 231-254.

Prache S., Thériez M., Béchet G., 1992. Complémentation des agneaux au pâturage pendant la phase d'allaitement. Interaction entre le niveau de complémentation et la quantité d'herbe offerte et effet sur le niveau de parasitisme. INRA Prod. Anim., 5, 137-148.

Priolo A., Micol D., Agabriel J., Prache S. Dransfield E., 2002. Effect of grass or concentrate feeding systems on lamb carcass and meat quality. Meat Sci., 62, 179-185.

Salette J., Lemaire G., 1981. Sur la variation de la teneur en azote des graminées fourragères pendant leur croissance : formulation d'une loi de dilution. C.R. Acad. Sci. Paris, 292, 875-878.

Salette J., Huché L., 1991. Diagnostic de l'état de nutrition minérale d'une prairie par l'analyse du végétal : principes, mise en oeuvre, exemples. Fourrages, 125, 3-18.

Signoret J.P., 1990. The influence of the ram effect on breeding activity of brebis and its underlying physiology. In: Reproductive physiology of Merino Sheep. Concepts and Consequences, Oldham C.M., Martin G.B. Purvis I.W. (Eds), School of Agriculture, The University of Western Australia, Nedlands, Perth, 59-70.

Speedy A.W., FitzSimons J., 1977. The reproductive performance of Finnish Landrace $x$ Dorset Horn and Border Leicester $x$ Scottish Blackface brebis mated three times in two years. Anim. Prod., 24, 189-196. 
Statistical Analysis System, 1999. SAS/STAT User's Guide, Version 8, SAS Institute Inc., Cary, NC.

Thériez M., 1983. Comportement alimentaire et ingestion de l'herbe par les brebis au pâturage. 8 èmes Journ. Rech. Ovine Caprine, 111-140.

Thériez M., Brelurut A., Pailleux J.Y., Benoit M., Liénard G., Louault F., De Montard F.X., 1997a. Extensification en élevage ovin viande par agrandissement des surfaces fourragères. Résultats zootechniques et économiques de 5 ans d'expérience dans le Massif Central Nord. INRA Prod. Anim., 10, 141-152.
Thériez M., Aurousseau B., Prache S. Mendizabal J., 1997b. Les défauts de couleur des gras d'agneaux. Renc. Rech. Rum., 4, 295-301.

Thimonier J., Cognié Y., Lassoued N., Khaldi G., 2000. L'effet mâle chez les ovins : une technique actuelle de maîtrise de la reproduction. INRA Prod. Anim., 13, 223-231.

Tournadre H., Bocquier F., Petit M., Thimonier J., Benoit M., 2002. Efficacité de l'effet bélier chez la brebis Limousine à différents moments de l'anoestrus saisonnier et selon la durée de l'intervalle tarissement-mise en lutte. Renc. Rech. Rum., 9, 143-146.
Tournadre H., Dulphy J.P., Jailler R., 2006. En agriculture biologique, réduire la part des concentrés dans la ration d'agneaux de bergerie sevrés : conséquences sur les quantités ingérées et les croissances. Renc. Rech. Rum., 13, 140.

Walrave Y., Cantin P., Desvignes A., Thimonier J., 1975. Variations saisonnières de l'activité sexuelle des races ovines du Massif Central. 1 ères Journ. Rech. Ovine Caprine, 261271

Yilma J. M., Dorchies P., 1991. Epidemiology of oestrus ovis in southwest France. Vet. Parasitol., 40, 315-323.

\title{
Résumé
}

Cette étude pluridisciplinaire de 4 ans réalisée en domaine expérimental a comparé deux systèmes d'élevage biologique d'ovins allaitants (118 brebis chacun), différant par le rythme de reproduction des brebis : une mise bas par brebis et par an (1/an), avec l'objectif d'une forte autonomie alimentaire vs 3 agnelages en 2 ans (3en2), avec l'objectif de maximiser le nombre d'agneaux produits par brebis. Les systèmes ont été évalués au travers du résultat économique, des performances et de la santé des animaux, ainsi que des niveaux d'autonomie fourragère et alimentaire. La productivité numérique par brebis a été en moyenne de $151 \%$ en $1 /$ an et de $161,3 \%$ en $3 e n 2$, avec une plus grande variabilité en 3en2. La consommation de concentré a été plus élevée en $3 e n 2$ qu'en 1/an $(+29 \%)$. La marge brute par brebis a été de $59 €$ en $3 e n 2$ vs $64 €$ en $1 / a n$. Le taux de mortalité des agneaux et le niveau de parasitisme ont été plus élevés en $3 e n 2$ qu'en $1 / a n$. La conformation des carcasses des agneaux, leur état d'engraissement et la couleur du gras de couverture ont été comparables entre les deux systèmes. Par contre, le poids de la carcasse et la fermeté du gras dorsal ont été inférieurs en 3en2 vs 1/an. L'intensification du rythme de reproduction n'a donc pas conduit à de meilleurs résultats économiques. La gestion du système $3 e n 2$ s'est révélée plus difficile, et ses résultats plus variables entre années. Au final, ce système d'élevage peut être considéré comme moins durable que le système classique non accéléré. Ce dernier a combiné des performances animales élevées et une meilleure autonomie alimentaire, facteurs déterminants des résultats technico-économiques, qui ont été, par ailleurs, supérieurs à ceux observés dans des fermes $\mathrm{AB}$ de la région.

\begin{abstract}
Comparison of two dairy sheep breeding systems with animals with different reproduction rhythms: a multidisciplinary experimental approach

A four-year interdisciplinary study was performed in an experimental farm to compare two organically managed sheep production systems (118 ewes each), differing in the ewes' reproduction rhythm: one lambing per ewe per year (1/year), with the aim of maximizing food self-sufficiency $v$ s. three lambings over two years (3in2), with the aim of maximizing ewe productivity. The farming systems were evaluated through economic results, animal health and performance, and forage and food self-sufficiency. Mean ewe productivity was $151 \%$ in $1 /$ year and $161,3 \%$ in 3 in 2 , with a higher between-year variability in the latter. Concentrate feed consumption per ewe was higher in 3 in 2 than in $1 /$ year $(+29 \%)$. Gross margin was $€ 59$ in $3 i n 2$ and $€ 64$ in 1/year. Lamb mortality and parasitism level were higher in 3 in 2 than in 1/year. Lamb carcass conformation, fatness and subcutaneous fat colour were not different between systems, but carcass weight and subcutaneous dorsal fat firmness were lower in $3 i n 2$ lambs than in 1/year. Intensification in an organically managed sheep system through an increased reproduction rhythm thus did not lead to better economic results and proved more variable and more difficult to manage, and thus less sustainable. The less intensive system (1/an) was both highly efficient from the animal standpoint and highly food self-sufficient. The technical and economic results of this system were higher than those of organic private farms in the same area.
\end{abstract}

BENOIT M., TOURNADRE H., DULPHY J.-P., LAIGNEL G., PRACHE S., CABARET J., 2009. Comparaison de deux systèmes d'élevage biologique d'ovins allaitants différant par le rythme de reproduction : une approche expérimentale pluridisciplinaire. Inra Prod. Anim., 22, 207-220. 